\title{
Gentzen Reduction Revisited
}

By

\author{
Mariko YASUGI*
}

\section{Introduction}

The major objective of this paper is to advocate Gentzen's first work on the consistency of arithmetic, which appeared in 1936 ([1]). He later published a new version of the consistency proof, and it is the reduction method of the latter which has been mostly used for various consistency proofs. His first work should be studied, however, more than it has been, not only for the strangely beautiful flavor it contains, but also for that it gives a general reduction method for arbitrary derivations (not just for supposedly contradictory ones), hence giving a systematic means to investigate the structure of formal systems of arithmetic.

We first present a reformulation of Gentzen's reduction of first order arithmetic in [1] in the style of [2] (\$1).

As an outright application of the result in Section 1, we obtain a form of quantifier-free interpretation of formal derivations of arithmetic (\$2) and an interpretation of derivable formulas $(\$ 3)$ in terms of ordinal recursive functions.

Variations of the preceding results will be given in Section 4 (first order systems) and in Section 5 (second order systems).

Many relevant studies have been made: those are seen, for example, in several papers of Kreisel, Schütte's book and Tait's paper (cf. [6] [12] and [14]). We wish to make the following points here. With our formulation, various known results follow systematically, without individual adjustments. It is also our point not to interpret a given system in a formal system with the (constructive) $\omega$-rule, for such a manoeuver seems to lose some delicate nature of the reducts of derivations.

Communicated by S. Takasu, February 26, 1977.

* Department of Mathematics, Faculty of Science, Shizuoka University.

This work is supported in part by NRC Grant \#A-3107 during my stay at Université de Montréal.

Present address; Institute of Information Science, University of Tsukuba, Sakuramura, Ibaraki, Japan 305. 
Technical terms and conventions have been adopted from [1], [2], [13] and [17], the knowledge of most of which is assumed in this article.

\section{§1. A Reformulation of Gentzen Reduction}

Definition 1.1. A formal system of arithmetic $\mathfrak{R}$ (cf. [1] and [2]).

1) Mathematical symbols: $0,{ }^{\prime},+, \cdot,=$.

2) Logical connectives: $>$ (not), $\wedge$ (and), $\forall$ (for all).

3) Free variables: $a_{1}, a_{2}, \cdots(a, b, c, \cdots)$.

4) Bound variables: $x_{1}, x_{2}, \cdots(x, y, z, \cdots)$.

5) Terms, formulas and sequents are defined from the symbols in 1) through 4) as usual.

6) Logical, initial sequents: $\mathfrak{D} \rightarrow \mathfrak{D}$, where $\mathfrak{D}$ is an arbitrary formula of $\mathfrak{N}$.

7) Mathematical, initial sequents (Mathematical axioms):

$$
\begin{aligned}
0=s^{\prime} & \rightarrow, \\
s=t & \rightarrow s^{\prime}=t^{\prime}, \\
s^{\prime}=t^{\prime} & \rightarrow s=t, \\
& \rightarrow s+0=s, \\
& \rightarrow s+t^{\prime}=(s+t)^{\prime}, \\
& \rightarrow s \cdot 0=0, \\
& \rightarrow s \cdot t^{\prime}=s \cdot t+s, \\
& \rightarrow s=s, \\
r=s, r=t & \rightarrow s=t,
\end{aligned}
$$

where $r, s$ and $t$ denote arbitrary terms.

8) The (formal) derivation of $\mathfrak{N}$ is formulated in the tree form as in [2], with the initial (topmost) sequents as defined in 6) and 7) above. In particular, the complete induction is formulated as below:

$$
\text { ind. } \frac{\mathfrak{F}(a), \Gamma \rightarrow \Theta, \mathfrak{F}\left(a^{\prime}\right)}{\mathfrak{F}(0), \Gamma \rightarrow \Theta, \mathfrak{F}(t)}
$$

where $a$ does not occur in $\mathfrak{F}(0), \Gamma$ and $\Theta$.

9) A term, a formula or a sequent which has no occurrences of free variables is said to be "closed." A derivation of $\mathfrak{N}$ is said to be closed if its endsequent is closed.

10) The endsequent of a derivation $P$ is often denoted by ends $(P)$.

11) A cut whose cut formula is atomic (equational) is called equational; a 
cut which is not equational is called essential.

12) An inference is called weak if it is a structural inference other than an essential cut; it is called strong otherwise.

13) A formula in a derivation $P$ is said to be explicit or implicit in $P$ according as the path of formulas containing it ends in the endsequent or as a cut formula. An inference is said to be explicit or implicit according as its principal formula is explicit or implicit.

Definition 1.2. Reduct-forms of a closed sequent. Let $S$ be a closed sequent and let $\Sigma$ be a set of closed sequents consisting of one, two or countably many sequents. $\quad \Sigma$ is called a reduct set of $S$ if it is related to $S$ according to one of the conditions listed below.

0) $\Sigma=\left\{S^{\prime}\right\}$ where every antecedent formula of $S^{\prime}$ is one of $S$ and every succedent formula of $S^{\prime}$ is one of $S$.

1) There is an occurrence of a formula $\mathfrak{Q}$ in the succedent of $S$ of the form $>\mathfrak{B}$, so $S$ is of the form

$$
\Gamma \rightarrow \Theta_{1},>\mathfrak{B}, \Theta_{2}
$$

and

$$
\Sigma=\left\{\mathfrak{B}, \Gamma \rightarrow \Theta_{1}, \mathfrak{N}, \Theta_{2}\right\} .
$$

2) $\mathfrak{U}:>\mathfrak{B}, S: \Gamma_{1}, \mathfrak{U}, \Gamma_{2} \rightarrow \Theta$ and $\Sigma=\left\{\Gamma_{1}, \mathfrak{U}, \Gamma_{2} \rightarrow \Theta, \mathfrak{B}\right\}$.

3) $\mathfrak{U}: \mathfrak{B} \wedge \mathfrak{E}, S: \Gamma \rightarrow \Theta_{1}, \mathfrak{A}, \Theta_{2}$ and $\Sigma=\left\{\Gamma \rightarrow \Theta_{1}, \mathfrak{B}, \mathfrak{A}, \Theta_{2} ; \Gamma \rightarrow \Theta_{1}, \mathfrak{E}, \mathfrak{A}, \Theta_{2}\right\}$.

4) $\mathfrak{A}: \mathfrak{B} \wedge \mathfrak{E}, S: \Gamma_{1}, \mathfrak{A}, \Gamma_{2} \rightarrow \Theta$ and either $\Sigma=\left\{\Gamma_{1}, \mathfrak{B}, \mathfrak{A}, \Gamma_{2} \rightarrow \Theta\right\}$ or $\Sigma=\left\{\Gamma_{1}\right.$, (c), $\left.\mathfrak{A}, \Gamma_{2} \rightarrow \Theta\right\}$.

5) $\mathfrak{U}: \forall x \mathfrak{F}(x), S: \Gamma \rightarrow \Theta_{1}, \mathfrak{N}, \Theta_{2}$ and $\Sigma=\left\{\Gamma \rightarrow \Theta_{1}, \mathfrak{F}(\boldsymbol{n}), \mathfrak{N}, \Theta_{2} ; n=0,1,2, \cdots\right\}$, where $n$ denotes the numeral expressing $n$.

6) $\mathfrak{A}: \forall x \mathfrak{F}(x), S: \Gamma_{1}, \mathfrak{A}, \Gamma_{2} \rightarrow \Theta$ and $\Sigma=\left\{\Gamma_{1}, \mathfrak{F}(s), \mathfrak{A}, \Gamma_{2} \rightarrow \Theta\right\}$ for some closed term $s$.

If $\Sigma$ is a reduct set of $S$, then we write $\Sigma_{s}$. We shall always assume that the members of a reduct set are enumerated in the order as shown in the definition.

If a closed sequent $S^{\prime}$ is in $\Sigma_{S}$, then we say that $S^{\prime}$ is an immediate (a first) reduct-form of $S$. We can define chains of immediate reduct-forms of $S$, thus evoking the definition of the reduct-forms of $S$ in general.

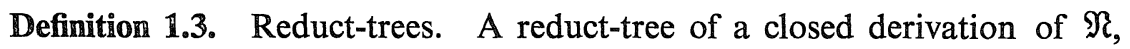
say $P$, is a tree with finite levels with zero, one, two or countably many nodes immediately above one node, having a closed derivation placed at each node 
and satisfying the following conditions.

(0) $P$ is placed at the bottom node (level 0 ) of the tree.

$(k) \rightarrow(k+1) \quad$ Let $Q$ be a derivation in the tree at the $k$ th level. Let $S$ be the endsequent of $Q$.

( $t$ ) If $Q$ consists of closed equations only, or $Q$ consists of a single logical, initial sequent, then there is no node above $Q$. We say in this case that $Q$ (or the node $Q$ sits on) is terminal.

Otherwise,

$(k+1)$ there are nodes above $Q$ (at level $k+1$ ). Let $\Pi_{Q}$ be the set of derivations above $Q$ with a specified enumeration, $Q_{0}, Q_{1}, \cdots, Q_{n}, \cdots, n<\alpha$ where $\alpha$ $=1,2$ or $\omega$. Then $\Sigma=\left\{\right.$ ends $\left(Q_{0}\right)$, ends $\left(Q_{1}\right), \cdots$, ends $\left.\left(Q_{n}\right), \cdots\right\}$ is a reduct-set of $S$, or $\Sigma=\Sigma_{s}$.

We say that each derivation in $\Pi_{Q}$ is immediately above $Q$.

A reduct-tree of $P$ will be denoted by $T_{P}$.

Branches and the partial orderings of nodes and branches of a tree can be defined in the usual manner. Other common notions such as "above," "below," "successor" and "predecessor" of nodes, and "extension," "restriction" etc. of branches will be used without defining them anew here.

Our task in Section 1 is to prove the following theorem.

Theorem 1. For any closed derivation of $\Re$, there is a reduct-tree associated with it which satisfies that every maximal, linear set of branches is finite. Namely, along any branch, there is a terminal derivation.

The consistency of $\mathfrak{N}$ follows immediately from the theorem.

The true significance of Theorem 1 is not the theorem as stated, but a particular construction of a reduct-tree, which is to be presented here.

It should be remarked also that the restriction of the theorem to closed derivations does not weaken the result: free variables in the endsequent can be bound without changing the content of the sequent.

Definition 1.4. The height of a sequent in a derivation is defined as in [2], and the ordinals below $\varepsilon_{0}$ are assigned to the derivations as in [2]; if $\alpha$ and $\beta$ are ordinals of the upper sequents of an equational cut, then the ordinal of the lower sequent is $\max (\alpha, \beta) . \quad o(S ; P)$ will denote the ordinal of $S$ a sequent in $P$ with regards to $P$, and $o(P)$ will denote the ordinal of $P$.

As an immediate consequence of the definition we have 
Corollary. $o(P)=1$ if and only if $P$ has no strong inferences.

Proof of Theorem 1. We are to present a uniform method to construct a tree $T_{P}$ for any closed $P$ so $T_{P}$ will satisfy the following conditions.

a) Suppose $Q_{2}$ is above $Q_{1}$ in $T_{P}$. Then $o\left(Q_{2}\right) \leq o\left(Q_{1}\right)$.

b) Suppose $Q_{1}$ is not terminal in $T_{P}$. If $o\left(Q_{1}\right)>1$, then along any branch passing $Q_{1}$ there is a $Q_{2}$ above $Q_{1}$ (at most three levels up) such that $o\left(Q_{2}\right)<o\left(Q_{1}\right)$. If $o\left(Q_{1}\right)=1$, then there are at most two levels above $Q_{1}$.

c) Every topmost derivation is terminal.

It is obvious that every branch has a finite extension whose topmost derivation is terminal. Therefore such a $T_{P}$ satisfies the requisite of Theorem 1 .

We shall first introduce a new rule of inference, term-replacement:

$$
\operatorname{tr} \frac{\Gamma \rightarrow \Theta}{\Gamma^{\prime} \rightarrow \Theta^{\prime}}
$$

where $\Gamma^{\prime} \rightarrow \Theta^{\prime}$ is obtained from $\Gamma \rightarrow \Theta$ by replacement of some occurrences of a closed term $s$ by another closed term $t$ where $s=t$ is true. A term-replacement is allowed only in the end-piece. It is regarded as a weak inference and the ordinal of the lower sequent is equal to that of the upper sequent. $(t)$ in Definition 1.3 will be modified so that the case where $Q$ consists of a logical initial sequent followed by some term-replacements be included.

For the construction of the tree, let us assume that we have defined the part of the tree to the $k$ th level and let $Q$ be a derivation at level $k$. In defining the immediate successors of $Q, \Pi_{Q}$, we closely follow Gentzen's reduction process in [2]. As we wish to avoid repetition, the reader should refer to [2]; see also [13] and [15].

$0^{\circ}$. $Q$ consists of closed equations, hence $Q$ is terminal. Stop. $Q$ has no successor.

$0^{*}$. We assume that $0^{\circ}$ is not the case.

$1^{\circ}$. Preliminary operation. If there are non-eigen free variables in the endpiece of $Q$, then replace them by 0 throughout. Put the resulting derivation $Q^{\prime}$ above $Q$. The endsequent and the ordinal remain unchanged. This takes only one step.

So, now,

$1^{*}$. we assume there is no non-eigen free variables in the end-piece of $Q$. $2^{\circ}$. The end-piece of $Q$ contains an ind. or an explicit logical inference. Let $I$ be a lowermost such.

2.1 . I is an ind. Do the "VJ-reduction" as in [2] and add a term-replacement: 


$$
\operatorname{tr} \frac{\mathfrak{F}(0), \Gamma \stackrel{\ddots}{\because} \underset{\rightarrow}{\rightarrow} \Theta, \mathfrak{F}(\boldsymbol{n})}{\mathfrak{F}(0), \Theta \rightarrow \Gamma, \mathfrak{F}(t)},
$$

where $t=\boldsymbol{n}$. Place the resulting $Q^{\prime}$ above $Q . o\left(Q^{\prime}\right)<o(Q)$ as proven in [2], and ends $\left(Q^{\prime}\right)=$ ends $(Q)$.

2.2 $2^{\circ} \quad I$ is $\forall$ in the succedent. Let $Q$ be of the form

$$
\begin{aligned}
& \frac{\because \because \cdot(a)}{\Gamma \stackrel{\alpha}{\longrightarrow} \Theta, \mathfrak{F}(a)} \\
& \Gamma \stackrel{\alpha+1}{\longrightarrow} \Theta, \forall x \mathfrak{F}(x) \\
& \Delta \stackrel{\beta}{\longrightarrow} \Lambda_{1}, \forall x \mathfrak{F}^{\prime}(x), \Lambda_{2},
\end{aligned}
$$

where $(a)$ indicates all the occurrences of $a$ in $Q, \mathfrak{F}$ and $\mathfrak{F}^{\prime}$ are identical up to term-replacements, the explicitly written $\forall x \mathfrak{F}^{\prime}(x)$ is a descendant of the explicitly written $\forall x \mathfrak{F}(x)$ and the Greek letter above a sequent denotes its ordinal with regards to $Q$.

Consider $Q_{n}$ for every $n, n=0,1,2, \cdots$ :

$$
\begin{aligned}
& \begin{aligned}
\Gamma \stackrel{\alpha}{\because}(\boldsymbol{n}) \\
\Gamma \stackrel{\alpha}{\longrightarrow} \mathfrak{F}(n), \Theta(n)
\end{aligned}
\end{aligned}
$$

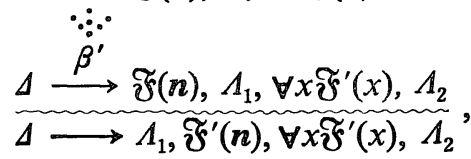

where $(\boldsymbol{n})$ indicates the replacement of $\alpha$ by $\boldsymbol{n}$ and a wavy line denotes omission of some weak inferences. $\beta^{\prime}<\beta$ can be easily established, and $o\left(Q_{n}\right)<o(Q)$ for every $n$. Place $\left\{Q_{n}\right\}_{n}$ above $Q$, or let $\Pi_{Q}$ be $\left\{Q_{0}, Q_{1}, \cdots, Q_{n}, \cdots\right\}$.

$2.3^{\circ}$. $I$ is $\forall$ in the antecedent. Let $Q$ be of the form

$$
\begin{aligned}
& \frac{\mathfrak{F}(t), \Gamma \stackrel{\because}{\rightarrow} \Theta}{\forall x \mathfrak{F}(x), \Gamma \rightarrow \Theta} \\
& \Delta_{1}, \forall x \mathfrak{F}^{\prime}(x), \stackrel{\because}{\because \bullet}, \stackrel{\Delta_{2}}{\rightarrow} \Lambda .
\end{aligned}
$$

Here $t$ is closed. Place the $Q^{\prime}$ defined below above $Q$ : 


$$
\begin{aligned}
& \frac{\mathfrak{F}(t), \Gamma \stackrel{\because}{\because} \rightarrow \Theta}{\forall x \mathfrak{F}(x), \Gamma, \mathfrak{F}(t) \rightarrow \Theta} \\
& \underbrace{\stackrel{\because}{\rightarrow}(x), \Delta_{2} \rightarrow \Lambda}_{\Delta_{1}, \widetilde{J}^{\prime}(t), \forall x \mathfrak{F}^{\prime}(x), \Delta_{2}, \mathfrak{F}^{\prime}(t)} .
\end{aligned}
$$

$2.4^{\circ} . \quad I$ is $\wedge$ in the succedent and $Q$ is of the form:

$$
\begin{aligned}
& \frac{\stackrel{\because}{\because} \rightarrow \Theta, \mathfrak{U}_{1} \quad \Gamma \stackrel{\because}{\rightarrow} \Theta, \mathfrak{A}_{2}}{\Gamma \rightarrow \Theta, \mathfrak{A}_{1} \wedge \mathfrak{U}_{2}} \\
& \Delta \stackrel{\because}{\rightarrow} \Lambda_{1}, \mathfrak{A}_{1}{ }^{\prime} \wedge \mathfrak{U}_{2}{ }^{\prime}, \Lambda_{2} \text {. }
\end{aligned}
$$

Let $Q_{i}$ be:

$$
\begin{aligned}
& \Gamma \stackrel{\because \because \bullet}{\rightarrow} \Theta, \mathfrak{N}_{i} \\
& \widetilde{\Gamma \rightarrow \mathfrak{U}_{i}}, \widetilde{\Theta}, \mathfrak{\mathfrak { U }}_{1} \wedge \mathfrak{\mathfrak { U }}_{2} \\
& \because \because \\
& \underset{\Delta \rightarrow \Lambda_{1}, \mathfrak{U}_{i}{ }^{\prime}, \mathfrak{U}_{1}{ }^{\prime} \wedge \mathfrak{P}_{2}{ }^{\prime}, \Lambda_{2}}{ }{ }^{\prime},
\end{aligned}
$$

for $i=1,2$. Let $\Pi_{Q}$ be $\left\{Q_{1}, Q_{2}\right\}$.

$2.5^{\circ}$. Other cases can be dealt with in a similar manner.

$2^{*}$. It will be assumed that there is no ind. or explicit logical inference in the end-piece of $Q$.

$3^{\circ}$. There is a weakening in the end-piece of $Q$. Elimination of weakenings from the end-piece of $Q$ be defined as in [2]. Let the resulting derivation $Q^{\prime}$ be the immediate successor of $Q$. $o\left(Q^{\prime}\right) \leq o(Q)$ and ends $\left(Q^{\prime}\right)$ is related to ends $(Q)$ according to 0 ) of Definition 1.2.

3*. There is no weakening in the end-piece of $Q$.

$4^{\circ}$. There is a logical initial sequent in the end-piece of $Q: \mathfrak{D} \rightarrow \mathfrak{D}$.

Suppose first both occurrences of $\mathfrak{D}$ are explicit. Since there are no ind.'s, logical inferences or weakenings in the end-piece of $Q, Q$ consists of $\mathfrak{D} \rightarrow \mathfrak{D}$ followed by some term-replacements. Stop. $Q$ is terminal.

Suppose next $Q$ is of the form:

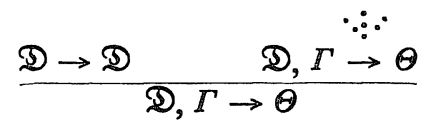

$$
\begin{aligned}
& \Delta \stackrel{\because}{\rightarrow} \Lambda .
\end{aligned}
$$


Define $Q^{\prime}$ :

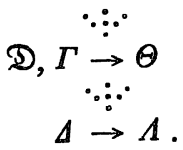

The other case is dealt with likewise.

4*. There is no logical, initial sequent in the end-piece of $Q$.

$5^{\circ}$. If $Q$ were its own end-piece, then $1^{*}$ through $4^{*}$ would enforce that $Q$ be terminal; in fact $Q$ would consist of closed equations only, contradicting $0^{*}$. Therefore let us assume that $Q$ properly contains its end-piece, viz., there is a logical inference in $Q$. (It is automatically implied that those logical inferences are above the end-piece.) The existence of a suitable cut is proved as in [2]. The reduction of a suitable cut is carried out exactly as in [2]. As a consequence ends $(Q)=$ ends $\left(Q^{\prime}\right)$ and $o\left(Q^{\prime}\right)<o(Q)$ where $Q^{\prime}$ is the reduct of $Q$. Place $Q^{\prime}$ above $Q$.

$1^{\circ}$ through $5^{\circ}$ exhaust all the possibilities. In the course of the reduction, each of the cases where $o(Q)=o\left(Q^{\prime}\right)\left(1^{\circ}\right.$ and $\left.3^{\circ}\right)$ takes only one step, hence at most two of such in succession. If $Q$ is not terminal and $o(Q)=1$, then either $1^{\circ}$ or $3^{\circ}$ applies, hence there can be at most two levels above $Q$. It is thus obvious that a) $\sim$ c) are satisfied.

Analyzing the construction of a reduct tree we have just given, we learn that it is primitive recursive: there is a primitive recursive enumeration of the derivations in the tree associated with a given derivation. Furthermore the construction is uniform in the closed derivations. More precisely, we have

Theorem 2. There is a primitive recursive function of two arguments, say $f$, such that if $p$ represents a closed derivation $P$ of $\mathfrak{N}$ and $n$ represents a branch $N$ of a tree, then $f(n, p)$ represents the derivation sitting at the top node of the branch $N$ in $T_{P}$, the reduct-tree defined as above.

We denote the Gödel number of $P$ by $\ulcorner P\urcorner$.

Outline of the proof. It is well-known that the following functions and predicates are primitive recursive.

$\operatorname{seq}(n): \quad n$ is a sequence number (representing a branch).

$n * i$ : the $i$ th extension of a branch $n$ to the next level.

$s g(i)$ : the signum function.

$\overline{s g}(i): 1-s g(i)$.

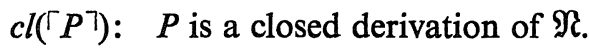


$\tau(\ulcorner Q\urcorner): Q$ is terminal.

The case conditions $0^{\circ}$ through $5^{\circ}$.

The assumptions $0^{*}$ through $4^{*}$.

$1^{\circ}$ applies to $Q$ and $r_{1}(\ulcorner Q\urcorner)=\left\ulcorner Q^{\prime}\right\urcorner$.

$2.1^{\circ}$ and $r_{2.1}\left(\left\ulcorner Q^{\top}\right)=\left\lceil Q^{\prime}\right\urcorner\right.$.

$2.2^{\circ}$ and $r_{2.2}(i,\ulcorner Q\urcorner)=\left\ulcorner Q_{i}\right\urcorner, i=0,1,2, \cdots$.

$2.4^{\circ}$ and $r_{2.4}(i,\ulcorner Q\urcorner)=\left\ulcorner Q_{i+1}\right\urcorner$, for $i=0,1$.

$2 . j^{\circ}$ applies to $Q$, where $j \neq 1,2,4$, and $r_{2, j}(\ulcorner Q\urcorner)=\left\ulcorner Q^{\prime}\right\rceil$, where $Q^{\prime}$ is the successor of $Q$ defined in $2 . j^{\circ}$.

For $l=3,4,5$, if $l^{0}$ applies to $Q$ and not $\tau(\ulcorner Q\urcorner)$, then $r_{l}(\ulcorner Q\urcorner)=\left\ulcorner Q^{\prime}\right\urcorner$ where $Q^{\prime}$ is defined in $l^{0}$.

Note that, in $2.1^{\circ},\lceil\boldsymbol{n}\rceil$ is a primitive recursive function of $\lceil t\rceil$, hence of $\lceil Q\urcorner$. We assume of course that those functions assume the value 0 when circumstances do not fit the described conditions.

Suppose now $\operatorname{seq}(n)$ and $\operatorname{cl}(\ulcorner P\urcorner)$ and define $f(n,\ulcorner P\urcorner)$ as follows.

$$
\begin{gathered}
f(n,\ulcorner P\urcorner)=\ulcorner P\urcorner \quad \text { if } n \text { represents the initial node of a tree; } \\
f(n * i,\ulcorner P\urcorner)=\left\{\begin{array}{c}
0 \quad \text { if } f(n,\ulcorner P\urcorner)=0 \text { or } \tau(f(n,\ulcorner P\urcorner)) ; \\
r_{l}(f(n,\ulcorner P\urcorner)) \cdot \overline{\operatorname{sg}(i)} \text { if } l \text { is one of } 1^{\circ} \sim 5^{\circ} \text { except } 2.2^{\circ} \text { and } \\
2.4^{\circ}, \text { and } l^{\circ} \text { applies to } f(n,\ulcorner P\urcorner) ; \\
r_{2, j}(i, f(n,\ulcorner P\urcorner)) \text { if } j=2 \text { or } 4 \text { and } 2.2^{\circ} \text { or } 2.4^{\circ} \text { applies to } \\
f(n,\ulcorner P\urcorner) .
\end{array}\right.
\end{gathered}
$$

In all other circumstances $f(n,\ulcorner P\urcorner)$ is defined to be 0 .

Note that $\ulcorner P\urcorner$ remains invariant throughout the definition and $f$ is defined by a primitive recursion (a course-of-values recursion) on $n$ ( $n$ as a natural number, not as a sequence).

Suppose $Q$ is in $T_{P}$ as defined above and $Q^{\prime}$ is an immediate successor of $Q$ in $T_{p}$. Let $S=e n d s(Q)$ and $S^{\prime}=e n d s\left(Q^{\prime}\right)$. Then $S^{\prime}$ is a reduct-form of $S$. But in this particular construction, we call $S^{\prime}$ a reduct of $S$. The difference is that, for 4) and 6) of Definition 1.2, a choice between $B$ and $C$ and a choice of a term $s$ respectively are specified here, and only then reduct-forms become meaningful; they are not sheer forms here.

\section{\$2. A Reduction to Quantifier-Free Derivations}

Definition 2.1. Positive and negative occurrences of quantifiers in a formula. 
By a quasi-subformula of a formula we mean a subformula-like expression which may have some free occurrences of bound variables. Let $\mathfrak{Y}$ be a formula of $\mathfrak{N}$, let be a quantifier in $\mathfrak{A}$ and let $\mathfrak{B}$ be a quasi-subformula of $\mathfrak{A}$ in which \# occurs. We shall define the positive occurrence or the negative occurrence of in $\mathfrak{B}$. Taking $\mathfrak{X}$ as $\mathfrak{B}$, we obtain the desired notion for $\mathfrak{A}$.

1) $\mathfrak{B}$ is $\forall x \mathfrak{F}(x)$ where the outermost $\forall x$ is $\#$. Then is positive in $\mathfrak{B}$.

2) $\mathfrak{B}$ is $\mathfrak{E} \wedge \mathfrak{D}$. negative in one of $\mathfrak{C}$ and $\mathfrak{D}$.

3) $\mathfrak{B}$ is $>\mathfrak{C}$. positive in $\mathbb{C}$.

4) $\mathfrak{B}$ is $\forall y \mathscr{S}(y)$ where $\#$ is not the outermost $\forall y$ in $\mathfrak{B}$. \# is positive or negative in $\mathfrak{B}$ according as it is positive or negative in $\mathbb{S}(y)$.

Definition 2.2. Positive forms and negative forms of a formula.

Let $\mathfrak{A}$ be a closed formula of $\mathfrak{N}$.

1) We first prepare a countable set of new symbols, which will be called eigenvariables and assumed to be ordered in $\omega$-type. A figure $\mathfrak{U}^{p}$ is obtained from $\mathfrak{X}$ by knocking off all the positive quantifiers in $\mathfrak{X}$ and then be replacing the remaining, corresponding bound variables by distinct eigenvariables. Those new variables will be called eigenvariables for $\mathfrak{A}$, or for an $\mathfrak{U}^{+}$, where $\mathfrak{X}^{+}$is to be defined subsequently. We do not specify which eigenvariables be used for $\mathfrak{U}$ for the time being. $\mathfrak{A}^{p}$ will again be called a formula.

2) We now prepare another countable set of new symbols, which will be called denotation-variables. They are assumed to be ordered in $\omega$-type. Suppose there are $m$ quantifiers in $\mathfrak{U}^{p}$. Let $\mathcal{A}_{1}, \mathcal{A}_{2}, \cdots, \mathcal{A}_{m}$ be a sequence of nonempty, finite sequences of denotation-variables such that the variables in them form a sequence of consecutive variables in its pre-determined order. Let $\mathcal{A}$ denote $\mathscr{A}_{1}, \mathscr{A}_{2}, \cdots, \mathscr{A}_{m}$. We shall define some quantifier-free forms of $\mathscr{A}$ relative to an assignment $\mathcal{A}$ of denotation-variables, which will be called the positive forms of $\mathfrak{A}$ relative to $\mathcal{A}$. We shall call $\mathcal{A}$ an assignment for $\mathfrak{A}$.

Let $\mathfrak{B}^{p}$ be any quasi-subformula of $\mathfrak{U}^{p}$. We shall define positive forms of $\mathfrak{B}^{p}$ relative to $\mathfrak{A}$ in a manner that, if $\mathfrak{B}^{+}$is a positive form of $\mathfrak{B}^{p}$, then $\mathfrak{B}^{+}$ is quantifier-free and a bound variable occurs (free) in $\mathfrak{B}^{+}$if and only if it occurs free in $\mathfrak{B}^{p}$, and there are only finitely many of positive forms of $\mathfrak{B}^{p}$ for a given $\mathcal{A}$.

(1) $\mathfrak{B}^{p}$ is atomic. Then $\mathfrak{B}^{p}$ is a sole positive form of $\mathfrak{B}^{p}$.

(2) $\mathfrak{B}^{p}$ is $7 \mathfrak{C}^{p}$. For any positive form of $\mathfrak{E}^{p}$, say $\mathfrak{F}^{+},>\mathfrak{C}^{+}$is a positive 
form of $\mathfrak{B}^{p}$.

(3) $\mathfrak{B}^{p}$ is $\mathfrak{C}^{p} \wedge \mathfrak{D}^{p}$. Let $C_{1}, \cdots, C_{l}$ be all the positive forms of $\mathfrak{C}^{p}$ (relative to $\mathcal{A})$ and let $D_{1}, \cdots, D_{r}$ be all the positive forms of $\mathfrak{D}^{p}$. Then $\left(C_{1} \vee \cdots \vee C_{l}\right)$ $\wedge\left(D_{1} \vee \cdots \vee D_{r}\right)$ is a sole positive form of $\mathfrak{B}^{p}$, where $A \vee B$ is short for $>(\neg A \wedge$ $>B)$.

(4) $\mathfrak{B}^{p}$ is $\forall x \mathfrak{F}(x)$. Let $x$ be the $i$ th bound variable in $\mathfrak{Q}^{p}$ (in its predetermined order). Let $F(x)$ be a positive form of $\mathfrak{F}(x)$ and let $\alpha$ be any denotationvariable from $\mathcal{A}_{1}$. Then $F(\alpha)$ is a positive form of $\mathfrak{B}^{p}$.

Now let $\mathfrak{U}^{p}$ be $\mathfrak{B}^{p}$. Then any positive form of $\mathfrak{U}^{p}$ is called a positive form of $\mathfrak{A}$ (relative to $\mathfrak{A}$ ). We may write $\mathfrak{A}^{+}$for a positive form of $\mathfrak{A}$; this does not specify a particular one. It is obvious from the definition that there are only finitely many positive forms of $\mathfrak{A}$ when an assignment $\mathcal{A}$ is specified. 3) We can define negative forms of $\mathfrak{X}$ in a like manner, reading "negative quantifiers" in the place of "positive quantifiers," $\mathfrak{U}^{n}, \mathfrak{B}^{n}$, etc. in the place of $\mathfrak{A}^{p}, \mathfrak{B}^{p}$, etc., and $\mathfrak{U}^{-}, \mathfrak{B}^{-}$, etc. in the place of $\mathfrak{A}^{+}, \mathfrak{B}^{+}$, etc. We use the same set of denotation-variables for negative forms as for positive forms.

Corollary. 1) In (3) of 2) in Definition 2.2, if a bound variable is shared by $a C_{i}$ and $a D_{j}$, then it is shared by all $C_{1}, \cdots, C_{m}, D_{1}, \cdots, D_{n}$.

2) Let $\mathfrak{U}^{+}$be a positive form of $\mathfrak{A}$ and let $\mathfrak{B}^{\prime}$ be the part of $\mathfrak{X}^{+}$corresponding to $\mathfrak{B}^{p}$. Then $\mathfrak{B}^{\prime}$ is obtained from $\mathfrak{B}^{+}$by replacing bound variables by appropriate denotation-variables. Therefore if (3) applies to $\mathfrak{B}^{+}$and $C_{1}{ }^{\prime}, \cdots, C_{m}{ }^{\prime}, D_{1}{ }^{\prime}, \cdots$, $D_{n}{ }^{\prime}$ correspond to $C_{1}, \cdots, C_{m}, D_{1}, \cdots, D_{n}$ respectively, then a denotation-variable which is shared by a $C_{i}{ }^{\prime}$ and $a D_{j}{ }^{\prime}$ is shared by all $C_{1}, \cdots, C_{m}, D_{1}, \cdots, D_{n}$.

The negative version of 2) also holds.

In passing, it is obvious that all the positive and negative forms of $\mathfrak{A}$ relative to an assignment $\mathcal{A}$ are decidable from $\mathfrak{U}$ and $\mathcal{A}$.

Definition 2.3. Let $S$ :

$$
\mathfrak{N}_{1}, \mathfrak{N}_{2}, \cdots, \mathfrak{N}_{m} \rightarrow \mathfrak{B}_{1}, \mathfrak{B}_{2}, \cdots, \mathfrak{B}_{n}
$$

be a closed sequent of $\mathfrak{R}$. Let $\mathcal{A}^{*}=\left(\mathcal{A}^{1}, \mathcal{A}^{2}, \cdots, \mathcal{A}^{m}\right)$ be an assignment of denotation-variables for the formulas $\mathfrak{A}_{1}, \mathfrak{A}_{2}, \cdots, \mathcal{A}_{m}$, and let $\mathscr{B}^{*}=\left(\mathcal{B}^{1}, \mathscr{B}^{2}, \cdots\right.$, $\mathscr{B}^{n}$ ) be an assignment for $\mathfrak{B}_{1}, \mathfrak{B}_{2}, \cdots, \mathfrak{B}_{n}$, where all of $\mathcal{A}^{1}, \mathcal{A}^{2}, \cdots, \mathcal{A}^{m}, \mathscr{B}^{1}, \mathscr{B}^{2}, \cdots$, $\mathscr{B}^{n}$ are mutually disjoint and the variables in $\left(\mathscr{A}^{*}, \mathscr{B}^{*}\right)$ form an initial segment of the sequence of denotation-variables in its order.

For each $i, 1 \leq i \leq m$, let $A_{i 1}, A_{i 2}, \cdots, A_{i p_{i}}$ be all the negative forms of $\mathfrak{U}_{i}$ 
relative to $\mathfrak{U}^{*}$, and for each $j, 1 \leq j \leq n$, let $B_{j 1}, B_{j 2}, \cdots, B_{j q_{j}}$ be all the positive forms of $\mathfrak{B}_{j}$ relative to $\mathscr{B}^{*}$, where the eigenvariables of those satisfy that $A_{i 1}$, $A_{i 2}, \cdots, A_{i p_{i}}$ share the same assignment of eigenvariables (for each negative quantifier in $\mathfrak{U}_{i}$ ); the same holds with $B_{j 1}, B_{j 2}, \cdots, B_{j q_{j}}$ for each positive quantifier in $\mathfrak{B}_{j}$; eigenvariables for different formulas are distinct, and the sequence of all the eigenvariables for $S$ (in an appropriate order) form an initial segment of the eigenvariables. Then

$$
\begin{gathered}
A_{11}, A_{12}, \cdots, A_{1 p_{1}}, \cdots, A_{i 1}, A_{i 2}, \cdots, A_{i p_{1}}, \cdots, \\
A_{m 1}, A_{m 2}, \cdots, A_{m p_{m}} \\
\rightarrow B_{11}, B_{12}, \cdots, B_{1 q_{1}}, \cdots, B_{j 1}, B_{j 2}, \cdots, B_{j q}, \cdots, \\
B_{n 1}, B_{n 2}, \cdots, B_{n q_{n}},
\end{gathered}
$$

or, for short,

$$
\begin{aligned}
& \left\{A_{i l}\right\}_{l=1, \cdots, p_{i}} ; i=1, \cdots, m \\
\rightarrow & \left\{B_{j l}\right\}_{l=1, \cdots, q_{j}} ; j=1, \cdots, n
\end{aligned}
$$

is called a quantifier-free form of $S$ relative to $\left(\mathcal{A}^{*}, \mathscr{B}^{*}\right)$, and will be denoted by $\left[\mathcal{A}^{*}, \mathscr{B}^{*}, S\right]$ or $[S]$ for short.

From the way $[S]$ was defined, it is obvious that the eigenvariables and the number of sets of the denotation-variables for $S$ are automatically determined from $S$, hence $[S]$ is decidable from $S$ and the number of variables in each of $\mathcal{A}^{i}$ and $\mathscr{B}^{j}$. For our purpose, names of variables are immaterial. Only identity and distinction of variables and the number of denotation-variables assigned to each relevant quantifier matter.

Proposition. We can arithmetize $\mathfrak{N}$ together with quantifier-free forms of closed sequents of $\mathfrak{N}$ in a manner that $[[\mathcal{A}, S]\urcorner$ is a primitive recursive function of $\ulcorner\mathcal{A}\urcorner$ and $\ulcorner S\urcorner$, where $\ulcorner X\urcorner$ denotes Gödel number of $X$ and $\mathcal{A}$ is an assignment for $S$ satisfying the condition in Definition 2.3.

Definition 2.4. Let $\alpha$ be an ordjnal below $\varepsilon_{0}$ and let $<^{\alpha}$ be (the arithmetization of) the canonical well-ordering of ordinals below $\alpha$. We assume 0 represents the least element of $<^{\infty}$, and $<^{\infty}$ will be abbreviated to $<$ when $\alpha$ is fixed. The class of $\alpha$-recursive functions is defined to be the minimal set of (number-theoretic) functions containing all the primitive recursive functions and closed under the " $\alpha$-recursion," which is defined below.

$$
f\left(0, x_{1}, \cdots, x_{n}\right)=h\left(x_{1}, \cdots, x_{n}\right) ;
$$




$$
\begin{aligned}
& f\left(x, x_{1}, \cdots, x_{n}\right) \\
= & g\left(x, x_{1}, \cdots, x_{n}, f\left(\tau_{1}^{*}\left(x, x_{1}, \cdots, x_{n}\right), x_{1}, \cdots, x_{n}\right),\right. \\
& \left.\cdots, f\left(\tau_{k}^{*}\left(x, x_{1}, \cdots, x_{n}\right), x_{1}, \cdots, x_{n}\right)\right)
\end{aligned}
$$

if $x>0$, where $\tau_{1}, \cdots, \tau_{k}$ are proper to $f . \tau^{*}$ is defined in general:

$$
\begin{aligned}
& \tau^{*}\left(x, x_{1}, \cdots, x_{n}\right) \\
= & \begin{cases}\tau\left(x, x_{1}, \cdots, x_{n}\right) & \text { if } \tau\left(x, x_{1}, \cdots, x_{n}\right)<x, \\
0 & \text { otherwise. }\end{cases}
\end{aligned}
$$

Note that when $\alpha$ is concretely given, $<$ is a primitive recursive predicate.

An $\alpha$-recursive function for any $\alpha$ below $\varepsilon_{0}$ is called an ordinal recursive function.

Theorem 3. Let $P$ be a closed derivation of $\mathfrak{N}$ and let $o(P)$ be $\theta$. Let $S_{0}=$ ends $(P)$ and let $\alpha=\omega^{\omega \theta+1}$. Then there is an $\alpha$-recursive function $\psi$ such that for any assignment of natural numbers to the eigenvariables for $S_{0}, \psi$ determines a quantifier-free form of $S_{0},\left[S_{0}\right]$, and some closed terms so that $S_{0}^{*}$, a closed, quantifier-free sequent of $\mathfrak{N}$ obtained from $\left[S_{0}\right]$ by replacing eigenvariables and denotation-variables by the numerals of the assigned numbers and the terms determined by $\psi$ respectively, is provable in $\mathfrak{N}$ without inferences of the ind. and the essential cut, hence without quantifiers (in fact the derivation is variablefree). $\psi$ also determines such a derivation. Precise requisites on $\psi$ will be described in the proof below.

Proof. We can define some primitive recursive functions and predicates of the following properties. (See also the proof of Theorem 2.)

$\operatorname{ct}(\ulcorner t\urcorner): t$ is a closed term.

$v(\ulcorner t\urcorner):$ the value of $t$ if $\operatorname{ct}(\ulcorner t\urcorner)$.

$l(n):$ the length of $n$ if $\operatorname{seq}(n)$.

$(n)_{i}$, where $0 \leq i \leq l(n)-1:$ the $i$ th entry of the sequence represented by $n$ if $\operatorname{seq}(n)$.

$\operatorname{cs}(\ulcorner S\urcorner): S$ is a closed sequent.

$a s(m,\ulcorner S\urcorner): m$ is an assignment of denotation-variables for $S$ if $\operatorname{cs}(\ulcorner S\urcorner)$.

$q f(q, m,\ulcorner S\urcorner): q$ is a quantifier-free form of $S$ relative to $m$ if $\operatorname{cs}(\ulcorner S\urcorner)$ and as $(m,\ulcorner S\urcorner)$. We may write this as $q=q f(m,\ulcorner S\urcorner)$.

$q f(\ulcorner T\urcorner): T$ is a quantifier-free sequent allowing the occurrences of eigenvariables and denotation-variables.

$e(\ulcorner S\urcorner)$ : the number of eigenvariables for $S$ if $c s(\ulcorner S\urcorner)$ or $q f(\ulcorner S\urcorner)$. 
$d([S]\urcorner):$ the number of denotation variables in $[S]$.

$\operatorname{cd}(\ulcorner Q\urcorner): Q$ is a closed derivation of $\Re$.

ends $(\ulcorner Q\urcorner)$ : the endsequent of $Q$ if $c d(\ulcorner Q\urcorner)$.

$c d^{*}(\ulcorner Q\urcorner): Q$ is a closed derivation of $\mathfrak{R}$ without ind.'s, essential cuts and quantifiers.

$S b\left(\ulcorner T\urcorner, n,\left(\left\ulcorner s_{1}\right\urcorner, \cdots,\left\ulcorner s_{l}\right\urcorner\right)\right)\left(=\left\ulcorner T^{*}\right\urcorner\right)$ : the result of substitution of numerals $\boldsymbol{n}_{1}, \cdots, \boldsymbol{n}_{r}$ for the eigenvariables of $T$ and of terms $s_{1}, \cdots, s_{l}$ for denotationvariables of $T$ when $q f(\ulcorner T\urcorner), r=e(\ulcorner T\urcorner), l=d(\ulcorner T\urcorner)$ and $n=\left(n_{1}, \cdots, n_{r}\right)$.

Now, the property $\psi$ has to satisfy is expressed in the condition $(C)$ below. (C) Let $r=e\left(\left\ulcorner S_{0}\right\urcorner\right)$. Suppose $\operatorname{seq}(n), l(n)=r$ and $n$ represents $\left(n_{1}, n_{2}, \cdots, n_{r}\right)$. If $\psi(n)=m$, then

(1) $\operatorname{seq}(m)$,

(2) $q f\left((m)_{0},(m)_{1},\left\ulcorner S_{0}\right\urcorner\right)$ and $l(m)-2=d\left(q f\left((m)_{1},\left\ulcorner S_{0}\right\urcorner\right)\right)$,

(3) $c t\left((m)_{i}\right)$, or $(m)_{i}=\left\ulcorner s_{i-1}\right\urcorner$ where $s_{i-1}$ is a closed term, $2 \leq i \leq l(m)-1$, and

(4) $c d^{*}\left((m)_{l(m)}\right)$, or $c d^{*}(\ulcorner R\urcorner)$ where $\ulcorner R\urcorner=(m)_{l(m)}$, and $S b\left(q f\left((m)_{1},\left\ulcorner S_{0}\right\urcorner\right), n, \sigma\right)$ $=\operatorname{end}(\ulcorner R\urcorner)$, where $\sigma=\left(s_{1}, \cdots, s_{l(m)-2}\right)$.

We construct $\psi$ via another $\alpha$-recursive function $\varphi(n, q)$.

(*) Let $P$ be as in the theorem and let $T_{P}$ be its reduct-tree. Let $f(q,\ulcorner P\urcorner)$ be the primitive recursive enumeration of $T_{P}$ (for all $P$ ) defined in Theorem 2 . We shall construct an $\alpha$-recursive function $\varphi(n, q)$ satisfying the following condition $\left(C^{\prime}\right)$.

$\left(C^{\prime}\right)$ Suppose $f(q,\ulcorner P\urcorner)=\ulcorner Q\urcorner$ (so $Q$ belongs to $T_{P}$ ). Let $S$ denote the endsequent of $Q$, or $\ulcorner S\urcorner=$ ends $(\ulcorner Q\urcorner)$. Changing $P$ to $Q$ and $S_{0}$ to $S$ in $(C)$, if we suppose $\varphi(n, q)=m$, then (1) (4) in (C) hold for $n, m, S$ and $Q$ for a fixed $P$.

If we let $Q$ be $P$, then we obtain $\psi: \psi(n)=\varphi(n,(0))$, where $(0)$ represents the bottom node of a tree.

For the construction of $\varphi$, still further primitive recursive functions and predicates are necessary. $\alpha\left(=\omega^{\omega^{\theta+1}}\right)$.

$<$ : (arithmetization of) the canonical ordering of ordinals below

$\varepsilon(\ulcorner Q\urcorner)$ : the number of free variables in the end-piece of $Q$ if $\operatorname{cd}(\ulcorner Q\urcorner)$.

$w(q)$ : the number of weakenings in the end-piece of $Q$.

$\mu(z)$ : (Gödel number of)

$$
\omega^{\omega 0(Q)+\varepsilon(\ulcorner Q\urcorner)}+\omega \cdot w(\ulcorner Q\urcorner)+n
$$

if $\ulcorner Q\urcorner=f(q,\ulcorner P\urcorner)$ and $z=(n, q)$.

Note that if $Q$ is in $T_{P}$, then 


$$
\begin{gathered}
\omega^{\omega^{\circ}(Q)+\varepsilon(\ulcorner Q\urcorner)}+\omega \cdot w(\ulcorner Q\urcorner)+n<\alpha . \\
(n, q)<(m, p): \mu((n, q))<\mu((m, p)) .
\end{gathered}
$$

$\varphi$ will be in its essence defined on $\mu(z)$. Namely, we shall define a function $\varphi(z)$ so that $\varphi(z)=\varphi(n, q)$ when $z$ represents the sequence $(n, q)$. We shall often write $\varphi(n, q)$ for $\varphi(z)$.

$g(n)$ : Gödel number of numeral $n$ (as a function of $n$ ). We also write this as $\lceil n\urcorner$.

$i(n, q): f(q,\ulcorner P\urcorner)=\ulcorner Q\urcorner, \quad n$ is an assignment to the eigenvariables of ends $(Q), 2.2^{\circ}$ applies to $Q$, and $i(n, q)$ is the entry of $n$ which is assigned to the explicitly written $\forall x$ in $\forall x \mathfrak{F}(x)$.

$\alpha(n, q)$ : the conditions as above hold, $n=\left(n_{1}, i(n, q), n_{2}, n_{3}\right)$ where $n_{1}$, $i(n, q), n_{2}, n_{3}$ are assignments to the eigenvariables of $\left(\Delta, \Lambda_{1}\right), \forall x, \mathfrak{F}(x), \Lambda_{2}$, respectively, and

$$
\alpha(n, q)=\left(n_{1}, n_{2}, i(n, q), n_{2}, n_{3}\right) .
$$

$\beta(n, q): f(q,\ulcorner P\urcorner)=\ulcorner Q\urcorner, 2.3^{\circ}$ applies to $Q, n=\left(n_{1}, n_{2}, n_{3}\right)$ where $n_{1}, n_{2}$ and $n_{3}$ respectively are assignments to the eigenvariables of $\Delta_{1}, \forall x \mathfrak{F}(x)$ and $\left(\Delta_{2}, \Lambda\right)$, and

$$
\beta(n, q)=\left(n_{1}, n_{2}, n_{2}, n_{3}\right) .
$$

$r_{i}(n, q), i=1,2: f(q,\ulcorner P\urcorner)=\ulcorner Q\urcorner, 2.4^{\circ}$ applies to $Q$ and $\gamma_{i}(n, q)$ is the assignment to the eigenvariables of ends $\left(Q_{i}\right)$ induced from $n$.

$\delta(n, q): 3^{\circ}$ applies and $\delta(n, q)$ is the assignment to ends $\left(Q^{\prime}\right)$ induced from $n$.

Similar functions can be defined for $5^{\circ}$.

$$
\tau_{1}(n, q)=\left\{\begin{array}{l}
(n, q * 0) \text { if } 1^{\circ} \text { or } 2.1^{\circ} \\
\text { applies to } f(q,\ulcorner P\urcorner)=\ulcorner Q\urcorner ; \\
(\alpha(n, q), q * i(n, q)) \text { if } 2.2^{\circ} ; \\
(\beta(n, q), q * 0) \text { if } 2.3^{\circ} \text { or } 2.5^{\circ} ; \\
\left(\gamma_{1}(n, q), q * 0\right) \text { if } 2.4^{\circ} \text { (and } Q_{1} \text { is taken); } \\
(\delta(n, q), q * 0) \text { if } 3^{\circ} ; \\
(n, q * 0) \text { if } 4^{\circ} \text { or } 5^{\circ}
\end{array}\right.
$$

$\tau_{2}(n, q)=\left(r_{2}(n, q), q * 1\right)$ if $2.4^{\circ}$ (and $Q_{2}$ is taken).

With all this at our disposal, we are to find out a primitive recursive scheme II so 


$$
\varphi(n, q)=\Pi\left(n, q, \varphi\left(\tau_{1}^{*}(n, q)\right), \varphi\left(\tau_{2}^{*}(n, q)\right)\right) .
$$

Define $\varphi(n, q)=0$ if $f(q,\ulcorner P\urcorner)=0$. Suppose $f(q,\ulcorner P\urcorner) \neq 0$. Then we go over the reduction process (the construction of $T_{P}$ ) defined in Section 1. The case descriptions there, $0^{\circ} \sim 5^{\circ}$, are primitive recursive (as a function of $q$ ). We shall constantly write $\ulcorner Q\urcorner$ for $f(q,\ulcorner P\urcorner)$.

$0^{\circ}$. $Q$ consists of closed equations, hence no need to define terms.

$1^{\circ}$. Substitution of 0 . Since the end-sequent remains unchanged, the induction hypothesis applies:

If

$$
\varphi(n, q)=\varphi(n, q * 0)=\varphi\left(\tau_{1}^{*}(n, q)\right) .
$$

then

$$
\mu(n, q)=\omega^{\omega^{0}(Q)+\varepsilon(\ulcorner Q\urcorner)}+\omega \cdot w(\ulcorner Q\urcorner)+n,
$$

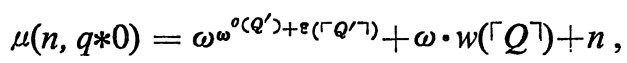

where $o(Q)=o\left(Q^{\prime}\right)$ and $\varepsilon\left(\left\ulcorner Q^{\prime}\right\urcorner\right)<\varepsilon(\ulcorner Q\urcorner)$. So $\mu(n, q * 0)<\mu(n, q)$, or $(n, q * 0)<(n, q)$, and hence $\tau_{1}^{*}(n, q)=\tau_{1}(n, q)$.

2.1 . $V J$-reduction. ends $\left(Q^{\prime}\right)=$ ends $(Q)$.

Define

$$
\varphi(n, q)=\varphi(n, q * 0)=\varphi\left(\tau_{1}^{*}(n, q)\right) .
$$

$o\left(Q^{\prime}\right)<o(Q)$, so $(n, q * 0)<(n, q)$.

2.2 $2^{\circ} \quad \forall$ in the succedent. Let $S$ denote ends $(Q)$ and let $S_{i}$ denote ends $\left(Q_{i}\right)$. We may identify $\mathfrak{F}$ and $\mathfrak{F}^{\prime}$ without loss of generality. Take the same assignment of numbers for $S_{i}$ as for $S$; this means the eigenvariables of $\mathfrak{F}(i)$ receives the assignment to the corresponding ones of $\mathfrak{F}(x)$. Thus, if the original assignment is $n$, then the resulting one is $\alpha(n, q) . \quad(\alpha(n, q), q * i(n, q))=\tau_{1}(n, q)$ $\left\langle(n, q)\right.$ since $o\left(Q_{i}\right)<o(Q)$. Therefore, for $\alpha(n, q)$ and $i=i(n, q)$, there is a quantifier-free form of $S_{i}$,

$$
\left[S_{i}\right]: \Delta^{*} \rightarrow \Lambda_{1}^{*},(\mathfrak{F}(i))^{*},(\forall x \Im(x))^{*}, \Lambda_{2}^{*} .
$$

It can be easily shown that if $F$ is a positive form of $\mathfrak{F}(\boldsymbol{i})$, then the occurrences of $i$ in $F$ corresponding to the indicated $i$ in $\mathfrak{F}(i)$ can be located. From this fact, $F(b)$, the expression obtained from $F$ by replacing those occurrences of $i$ by $b$, serves as a positive form of $\mathfrak{\Im}(b)$, where $b$ is the eigenvariable for the $\forall x$ in $\forall x \mathfrak{F}(x)$ in $(\forall x \mathfrak{F}(x))^{p}$. So, if

$$
(F(i))^{*}: F_{1}(i), \cdots, F_{k}(i)
$$

then

$$
[S]: \Delta^{*} \rightarrow \Lambda_{1}^{*}, F_{1}(b), \cdots, F_{k}(b),(\forall x \mathfrak{F}(x))^{* \prime}, \Lambda_{2}^{* \prime}
$$


where' means some change in the names of eigenvariables, can serve as a quantifier-free form of $S$. This process of transpassing from $\left[S_{i}\right]$ to $[S]$ is primitive recursive in $S$ and $\left[S_{i}\right]$. Let $S_{i}^{*}$ be the result of substitution of $\alpha(n, q)$ for the eigenvariables and the terms determined by $\varphi(\alpha(n, q), q * i(n, q))$ for the denotation variables in $\left[S_{i}\right]$. It has a derivation of the desired sort. $S^{*}$ obtained from $[S]$ by replacing eigenvariables by $\pi$ (numeral of $i(n, q)$ for $b$ ) and denotation-variables by the terms determined by $\varphi(\alpha(n, q), q * i(n, q))$, is identical with $S_{i}{ }^{*}$. So we can take the same derivation. Thus,

$$
\varphi(n, q)=\Pi_{1}(n, q, \varphi(\alpha(n, q), q * i(n, q)))
$$

where $\Pi_{1}(n, q, m)$ specifies $[S]$, some terms and a derivation depending on $n, q$ and $m$.

$$
\tau_{1}(n, q)=(\alpha(n, q), q * i(n, q))<(n, q)
$$

hence $\quad \varphi(n, q)=\Pi_{1}\left(n, q, \varphi\left(\tau_{1}^{*}(n, q)\right)\right)$.

2.3 . $\quad \forall$ in the antecedent. Let $S$ denote ends $(Q)$ and let $S^{\prime}$ denote ends $\left(Q^{\prime}\right)$. $[S]$ can be induced from $\left[S^{\prime}\right]$ as follows. Suppose

$$
\left[S^{\prime}\right]: \Delta_{1}^{*},(\mathfrak{F}(t))^{*},(\forall x \mathfrak{\oiint}(x))^{*}, \Delta_{2}^{*} \rightarrow \Lambda^{*} .
$$

Let $(\mathfrak{J}(t))^{*}$ be $F_{1}(t), F_{2}(t), \cdots, F_{k}(t)$, where $t$ indicates all the occurrences of $t$ corresponding to those in $\mathfrak{F}(t)$. Then

$$
\begin{gathered}
{[S]: \Delta_{1}^{*}, F_{1}\left(c_{1}\right), F_{2}\left(c_{2}\right), \cdots, F_{k}\left(c_{k}\right),(\forall x \mathfrak{F}(x))^{* \prime},} \\
\Delta_{2}{ }^{* \prime} \rightarrow \Lambda^{* \prime},
\end{gathered}
$$

where ' means some changes in the names of denotation-variables and $c_{1}$, $c_{2}, \cdots, c_{k}$ are some appropriate denotation-variables, can be a quantifier-free form of $S$. If we assign $t$ to $c_{1}, \cdots, c_{k}$ and retain all other terms for denotationvariables, $S^{*}=S^{* *}$ can serve as the desired sequence for $S$ and $n$. So, for a primitive recursive $\Pi_{2}$,

$$
\begin{gathered}
\tau_{1}(n, q)=\tau_{1}^{*}(n, q)=(\beta(n, q), q * 0)<(n, q) ; \\
\varphi(n, q)=\Pi_{2}\left(n, q, \varphi\left(\tau_{1}^{*}(n, q)\right)\right) .
\end{gathered}
$$

2.4 $4^{\circ} \wedge$ in the succedent. Let $S, S_{1}$ and $S_{2}$ denote ends $(Q)$, ends $\left(Q_{1}\right)$ and ends $\left(Q_{2}\right)$ respectively. There is a quantifier-free form of $S_{1},\left[S_{1}\right]$, and an assignment of terms determined by $r_{1}(n, q)$. With $S_{2}$ and $r_{2}(n, q)$ likewise.

Let

$$
\left[S_{1}\right]: \Delta^{*} \rightarrow \Lambda_{1}^{*}, A_{11}^{*}, \cdots, A_{1 k}^{*},\left(\mathfrak{U}_{1} \wedge \mathfrak{U}_{2}\right)^{*}, \Lambda_{2}^{*}
$$


and

$\left[S_{2}\right]: A^{* *} \rightarrow \Lambda_{1}^{* *}, A_{21}^{*}, \cdots, A_{2 l}^{*},\left(\mathfrak{U}_{1} \wedge \mathfrak{U}_{2}\right)^{* *}, \Lambda_{2}^{* *}$.

Consider

$$
\Delta^{*}, \Delta^{* *} \rightarrow \Lambda_{1}^{*}, \Lambda_{1}^{* *},\left(\bigvee_{i=1}^{k} A_{1 i}^{*}\right) \wedge\left(\bigvee_{j=1}^{l} A_{2 j}{ }^{*}\right),\left(\mathfrak{U}_{1} \wedge \mathfrak{U}_{2}\right)^{*},\left(\mathfrak{U}_{1} \wedge \mathfrak{U}_{2}\right)^{* *}, \Lambda_{2}^{*}, \Lambda_{2}^{* *}
$$

By rearranging the order of formulas and renaming variables in an appropriate manner, we obtain from the sequent immediately above,

$$
[S]: \widetilde{\Delta} \rightarrow \tilde{\Lambda}_{1},\left(\bigvee_{i=1}^{k} \tilde{A}_{1 i}\right) \wedge\left(\bigvee_{j=1}^{l} \tilde{A}_{2 j}\right),\left(\mathfrak{Q}_{1} \wedge \mathfrak{U}_{2}\right)^{\sim}, \tilde{\Lambda}_{2},
$$

which can serve as a quantifier-free form of $S$. An assignment of terms to denotation-variables in $[S]$ can be induced from those for $\left[S_{1}\right]$ and $\left[S_{2}\right]$. Let

and

$$
S_{1}{ }^{*}: \Delta^{\prime} \rightarrow \Lambda_{1}{ }^{\prime}, A_{11}{ }^{\prime}, \cdots, A_{1 k}{ }^{\prime},\left(\mathfrak{U}_{1} \wedge \mathfrak{U}_{2}\right)^{\prime}, \Lambda_{2}{ }^{\prime}
$$

$$
S_{2}{ }^{*}: \Delta^{\prime \prime} \rightarrow \Lambda_{1}^{\prime \prime}, A_{21}{ }^{\prime \prime}, \cdots, A_{2 l}{ }^{\prime \prime},\left(\mathfrak{\Re}_{1} \wedge \mathfrak{U}_{2}\right)^{\prime \prime}, \Lambda_{2}^{\prime \prime}
$$

be the instantiations of $\left[S_{1}\right]$ and $\left[S_{2}\right]$ respectively by $n_{1}=r_{1}(n, q), n_{2}=r_{2}(n, q)$ and the terms hereby determined.

$$
\left(n_{1}, q * 0\right)<(n, q),
$$

and

$$
\left(n_{2}, q * 1\right)<(n, q),
$$

hence $\tau_{1}{ }^{*}(n, q)=\tau_{1}(n, q)$ and $\tau_{2}{ }^{*}(n, q)=\tau_{2}(n, q)$, and there are derivations of $S_{1}{ }^{*}$ and $S_{2}{ }^{*}$, say $P_{1}$ and $P_{2}$ respectively, of the desired sort. Consider $P_{1}^{\prime}$ :

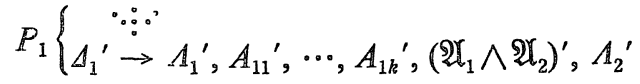

$$
\begin{aligned}
& \Delta^{\prime},{A^{\prime \prime}}^{\prime \prime} \Lambda_{1}^{\prime}, \Lambda_{1}^{\prime \prime},\left(\mathscr{U}_{1} \wedge \mathfrak{X}_{2}\right)^{\prime},\left(\mathfrak{\Re}_{1} \wedge \mathfrak{Q}_{2}\right)^{\prime \prime}, \Lambda_{2}^{\prime}, \Lambda_{2}^{\prime \prime}, A_{11}^{\prime}, \cdots, A_{1 k}{ }^{\prime} \\
& \Delta^{\prime}, \Delta^{\prime \prime} \rightarrow \Lambda_{1}^{\prime}, \Lambda_{1}^{\prime \prime},\left(\mathscr{U}_{1} \wedge \mathfrak{U}_{2}\right)^{\prime},\left(\mathfrak{U}_{1} \wedge \mathfrak{U}_{2}\right)^{\prime \prime}, \Lambda_{2}{ }^{\prime}, \Lambda_{2}^{\prime \prime}, \bigvee_{i=1}^{k} A_{1 i}{ }^{\prime}
\end{aligned}
$$

Similarly we obtain $P_{2}^{\prime}$ whose endsequent is

$$
\Delta^{\prime}, \Delta^{\prime \prime} \rightarrow \Lambda_{1}^{\prime}, \Lambda_{1}^{\prime \prime},\left(\mathfrak{U}_{1} \wedge \mathfrak{U}_{2}\right)^{\prime},\left(\mathfrak{U}_{1} \wedge \mathfrak{U}_{2}\right)^{\prime \prime}, \Lambda_{2}{ }^{\prime}, \Lambda_{2}^{\prime \prime}, \bigvee_{j=1}^{l} A_{2 j}{ }^{\prime \prime}
$$

Applying $\wedge$ in the succedent to the endsequents of $P_{1}{ }^{\prime}$ and $P_{2}{ }^{\prime}$, we obtain a derivation of the sequent:

$$
\Delta^{\dagger} \rightarrow \Lambda_{1}^{\dagger},\left(\bigvee_{i=1}^{k} A_{1 i}^{\dagger}\right) \wedge\left(\bigvee_{j=1}^{l} A_{2 j}^{\dagger}\right),\left(\mathfrak{U}_{1} \wedge \mathfrak{U}_{2}\right)^{\dagger}, \Lambda_{2}^{\dagger},
$$

which is the instantiation of $[S]$ with regards to $n$ and the terms which have been determined by $n_{1}$ and $n_{2}$ for $\left[S_{1}\right]$ and $\left[S_{2}\right]$ respectively. So for a primitive recursive $I_{3}$, 


$$
\varphi(n, q)=\Pi_{3}\left(n, q, \varphi\left(\tau_{1}^{*}(n, q)\right), \varphi\left(\tau_{2}^{*}(n, q)\right)\right) .
$$

2.5.$\wedge$ in the antecedent. Suppose a variable-free sequent of the form

$$
S^{*}: \Delta_{1}^{*},(\mathfrak{Y} \wedge \mathfrak{B})^{*}, A_{1}, \cdots, A_{m}, \Delta_{2}^{*} \rightarrow \Lambda^{*}
$$

and a derivation of $S^{*}$ of the desired sort have been obtained. Take the singleton assignment of denotation-vairables for $B$ and assign the term 0 to each denotation-variable to obtain $B$. Consider:

$$
\Delta_{1}^{*},(\mathfrak{Y} \wedge \mathfrak{B})^{*}, A_{1} \wedge B, \cdots, A_{n b} \wedge B_{2} L_{2}^{*} \stackrel{\mathfrak{S}^{*}}{\rightarrow} \Lambda^{*} .
$$

Other cases of $2.5^{\circ}$ can be dealt with in a similar manner.

$3^{\circ}$. Elimination of weakenings. $o\left(Q^{\prime}\right) \leq o(Q), \varepsilon\left(\left\ulcorner Q^{\prime}\right\urcorner\right) \leq \varepsilon(\ulcorner Q\urcorner)$ and $w\left(\left\ulcorner Q^{\prime}\right\urcorner\right)<$ $w(\ulcorner Q\urcorner)$. Thus, $(\delta(n, q), q * 0)<(n, q)$. There are an assignment and derivation desired for $Q^{\prime}$. For the formulas in ends $(Q)$ which do not occur in ends $\left(Q^{\prime}\right)$, take the singleton assignment of denotation-variables and assign 0 to every such denotation-variable. A desired derivation can be obtained from one for $Q^{\prime}$ by adding some weakenings at the end if necessary.

$4^{\circ}$. $Q$ consists of $\mathfrak{D} \rightarrow \mathfrak{D}$ alone. Take the singleton assignment to denotationvariables, so $[S]$ will have the form

$$
D(a, b) \rightarrow D(c, d)
$$

where $b$ and $c$ denote eigenvariables, and $a$ and $b$ denote denotation-variables. Let $n$ be $\left(n_{1}, n_{2}\right)$, where $n_{1}$ is assigned to $b$ while $n_{2}$ is assigned to $c$. Then assign $n_{2}$ to $a$ and assign $n_{1}$ to $d$, to obtain a logical, initial sequent. Here $\varphi$ is directly defined.

As for other cases of $4^{\circ}$ and $5^{\circ}$, since the endsequents remain unchanged, the induction hypothesis itself can serve as $\varphi(n, d)$ :

$$
\begin{aligned}
& \varphi(n, q)=\varphi(n, q * 0) . \\
& (n, q * 0)<(n, q) \text { since } o\left(Q^{\prime}\right)<o(Q) .
\end{aligned}
$$

$5^{\circ} . \quad(n, q * 0)<(n, q)$ and the endsequent remains the same. So

$$
\varphi(n, q)=\varphi\left(\tau_{1}^{*}(n, q)\right)
$$

Summing up the construction of $\varphi$ for all the cases, we can easily see that a primitive recursive $I I$ as desired can be defined. 


\section{$\S 3 . \quad$ An Interpretation of Arithmetic}

As an application of our theorem in the preceding section, we shall show how to interpret the system of arithemetic in terms of ordinal recursive functions. The result is originally due to Kreisel: see [7].

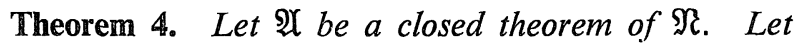

$$
A: A\left(c_{1}, \cdots, c_{q}, a_{1}, \cdots, a_{p}\right)
$$

be the positive form of $\mathfrak{X}$ with the singleton assignment of denotation-variables to negative quantifiers; $c_{1}, \cdots, c_{q}$ is the list of the denotation-variables in $A$ and $a_{1}, \cdots, a_{p}$ be the list of eigen-variables in $A$. Then, for some ordinal $\alpha$ below $\varepsilon_{0}$, there are $\alpha$-recursive functions of p-arguments, say $\varphi_{1}, \cdots, \varphi_{q}$, such that for any $p$ tuple of numbers $\left(n_{1}, \cdots, n_{p}\right)$ A becomes a true statement of arithmetic under the assignment,

$$
\begin{aligned}
& a_{1}: n_{1}, \cdots, a_{p}: n_{p}, \\
& c_{1}: \varphi_{1}\left(n_{1}, \cdots, n_{p}\right), \cdots, c_{q}: \varphi_{q}\left(n_{1}, \cdots, n_{p}\right) .
\end{aligned}
$$

We may express the theorem as follows:

$$
\exists f_{1} \cdots \exists f_{q} \forall x_{1} \cdots \forall x_{p} A\left(f_{1}\left(x_{1}, \cdots, x_{p}\right), \cdots, f_{q}\left(x_{1}, \cdots, x_{p}\right), x_{1}, \cdots, x_{p}\right),
$$

where $\exists f_{1}, \cdots, \exists f_{q}$ are function quantifiers, has an interpretation with

$$
f_{1}: \varphi_{1}, \cdots, f_{q}: \varphi_{q} .
$$

We shall first prove the following

Proposition. Let $\chi$ be a primitive recursive characteristic function of the variable-free sequents of $\mathfrak{N}$. We shall also use $\chi$ to denote such a function for formulas. Let $P_{0}$ be a derivation of $\rightarrow \mathfrak{A}$ whose ordinal is $\theta$ and let $\alpha$ be $\omega^{\omega^{\theta+1}}$. For any $Q$ in $T_{P_{0}}$, we write $S$ for ends $(Q)$ and $(S)$ or $S(c, a)$ for the quantifier-free form of $S$ with regards to the singleton assignment (of denotation-variables). $\left(^{*}\right)$ There is an $\alpha$-recursive function $\varphi_{0}(n, q)$ satisfying the condition $(D)$ stated below.

(D) Let $r=e(\ulcorner S\urcorner)$. Suppose seq(n), $l(n)=r$ and $n$ represents $\left(n_{1}, n_{2}, \cdots, n_{r}\right)$. If $\varphi_{0}(n, q)=m$, then
(1) $\operatorname{seq}(m)$,
(2) $l(m)=d(\ulcorner S(c, a)\rceil)=l$
and (3) $\chi(\ulcorner S(\boldsymbol{m}, \boldsymbol{n})\rceil)=0$, 
where $m$ denotes $\left(m_{0}, m_{1}, \cdots, m_{l-1}\right)$.

$\ulcorner S(m, n)\urcorner$ is a primitive recursive function of $n, m$ and $q$.

Proof of Theorem 4. Assume for now that the proposition holds. Let $Q$ be $P_{0}$; hence $S$ is $\rightarrow \mathscr{U}$. For any $n=\left(n_{1}, \cdots, n_{p}\right)$, if $\varphi_{0}(n,(0))=m$, then $\chi(\ulcorner A(m, n)\rceil)$ $=0$; namely $A(c, a)$ is true under the assignment $a: n$ and $c: m$. Define

$$
\varphi_{i}\left(n_{1}, \cdots, n_{p}\right)=\left(\varphi_{0}(n,(0))_{i-1},\right.
$$

$1 \leq i \leq q . \quad \varphi_{i}$ is $\alpha$-recursive and with those $\varphi_{1}, \cdots, \varphi_{q}$, the theorem holds.

The proposition $\left(^{*}\right)$ is proved in a manner similar to the proof of Theorem 3. We shall follow it and sketch only some new aspects.

Define $\varphi_{0}(n, q)=0$ if $f\left(q,\left\ulcorner P_{0}\right\urcorner\right)=0$. Suppose $f\left(q,\left\ulcorner P_{0}\right\urcorner\right) \neq 0$.

$0^{\circ}$. It can be easily proved that $\chi\left({ }^{[} S^{]}\right)=0$ if $S=e n d s(Q)$ and $Q$ satisfies $0^{\circ}$. Let $\varphi_{0}(n, q)$ represent the empty sequence.

$1^{\circ}$ and $2.1^{\circ}$. The induction hypothesis applies.

$2.2^{\circ}$. Let $i$ denote $i(n, q)$ and $\tilde{n}_{i}$ denote $\alpha(n, q)$. If $\varphi_{0}\left(\tilde{n}_{i}, q * i\right)=m_{i}$, then

$$
\chi\left(\left\ulcorner S_{i}\left(m_{i}, \tilde{\nexists g}_{i}\right)\right\urcorner\right)=0 .
$$

$S_{i}\left(m_{i}, \tilde{n}_{i}\right)$ is of the form

$$
\Delta^{i} \rightarrow \Lambda_{1}^{i}, F^{i}(i), F_{0}^{i}(i), \Lambda_{2}^{i},
$$

where $\Delta^{i}$ for different $i$ 's differ only by the numerals substituted for denotationvariables; the same with other formulas. $i$ does not change except at the indicated places. $F^{i}(i)$ and $F_{0}^{i}(i)$ differ only by the numerals substituted for denotation-variables. $\varphi_{0}(n, q)$ is obtained from $m_{i}$ by replacing the numbers corresponding to $F^{i}(i)$ and $F_{0}^{i}(i)$ by $\rho(i)$, where $\rho$ is difined below, retaining other numbers invariant.

$\rho(i)=\bar{\chi}\left(\left\ulcorner F^{i}(i)\right\urcorner\right) \cdot m_{F}+\chi\left(\left\ulcorner F^{i}(i)\right\urcorner\right) \cdot m_{F_{0}}$, where $m_{F}$ is the sequence of numbers (from $m_{i}$ ) corresponding to the negative quantifiers of $\mathfrak{\mho}(i)$ and $m_{F_{0}}$ corresponds to those of $\forall x \mathfrak{F}(x) . \rho$ is primitive recursive in $f\left(q,\left\ulcorner P_{0}\right\urcorner\right)$ and $\varphi_{0}\left(\tilde{n}_{i}, q^{*} i\right)$, and hence $\varphi_{0}(n, q)$ is defined by

$$
\varphi_{0}(n, q)=\Pi\left(n, q, \varphi_{0}\left(\alpha(n, q), q^{*} i(n, q)\right)\right),
$$

or

$$
\varphi_{0}(n, q)=\Pi\left(n, q, \varphi_{0}\left(\tau_{1}^{*}(n, q)\right)\right)
$$

with a primitive recursive $\Pi$.

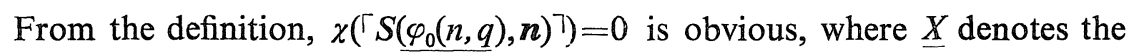


numeral of $X$.

$2.3^{\circ}$. Let $\tilde{n}$ denote $\beta(n, q)$. If $m=\varphi_{0}\left(\tilde{n}, q^{*} 0\right)$, then

$$
\chi\left(\left\ulcorner S^{\prime}(\boldsymbol{m}, \tilde{\boldsymbol{n}})\right\urcorner\right)=0 .
$$

$S^{\prime}(m, \tilde{n})$ is of the form

$$
\Delta_{1}^{\prime}, F^{\prime}(t), F_{0}^{\prime}(s), \Delta_{2}^{\prime} \rightarrow \Lambda^{\prime}
$$

$\varphi_{0}(n, q)$ is obtained from $m$ by replacing the numbers corresponding to $F^{\prime}(t)$ and $F_{0}^{\prime}(s)$ by $\rho$, which is defined below, retaining other numbers invariant.

$$
\rho=\chi\left(\left\ulcorner F^{\prime}(t)\right\urcorner\right) \cdot m_{F}+\bar{\chi}\left(\left\ulcorner F^{\prime}(t)\right\urcorner\right) \cdot m_{F_{0}},
$$

where $m_{F}$ is the sequence of numbers $(v(\ulcorner t\urcorner), \sigma), \sigma$ corresponding to the positive quantifiers of $F(t)$, and $m_{F_{0}}$ is the sequence of numbers $\left(s, \sigma_{0}\right), \sigma_{0}$ corresponding to the positive quantifiers of $\mathfrak{F}(x) . \quad \rho$ is primitive recursive in $f\left(q,\left\ulcorner P_{0}\right\urcorner\right)$ and $\varphi_{0}\left(\tilde{n}, q^{*} 0\right)$.

$2.4^{\circ}$. Consult $2.4^{\circ}$ in Section 2 for notations.

$$
\begin{aligned}
& \left(S_{1}\right): \Delta^{\prime} \rightarrow \Lambda_{1}^{\prime}, A_{1},\left(\mathfrak{A}_{1} \wedge \mathfrak{P}_{2}\right)^{\prime}, \Lambda_{2}^{\prime} \\
& \left(S_{2}\right): \Delta^{\prime \prime} \rightarrow \Lambda_{1}^{\prime \prime}, A_{2},\left(\mathfrak{A}_{1} \wedge \mathfrak{U}_{2}\right)^{\prime \prime}, \Lambda_{2}^{\prime \prime} .
\end{aligned}
$$

Suppose $\mathfrak{D}$ is in $\Delta$ and suppose $\sigma_{1}$ is the sequence of numbers corresponding to $\mathfrak{D}^{\prime}$, and $\sigma_{2}$ corresponding to $\mathfrak{D}^{\prime \prime}$. Define

$$
\sigma=\chi(\ulcorner\mathfrak{D}\urcorner) \cdot \sigma_{1}+\bar{\chi}\left(\left\ulcorner\mathfrak{D} \mathfrak{S}^{\prime}\right\urcorner\right) \cdot \sigma_{2} .
$$

If $\mathfrak{T}$ is in $\Lambda_{1}$ or $\Lambda_{2}$, define $\sigma$ by

$$
\sigma=\bar{\chi}\left(\left\ulcorner\mathfrak{S}^{\prime}\right\urcorner\right) \cdot \sigma_{1}+\chi\left(\left[\mathfrak{D}^{\prime}\right\urcorner\right) \cdot \sigma_{2} .
$$

If $\mathfrak{T}$ is $\mathfrak{U}_{1} \wedge \mathfrak{A}_{2}$, and if $\sigma_{1}, \sigma_{2}, \sigma_{3}$ respectively correspond to $A_{1} \wedge A_{2},\left(\mathfrak{U}_{1} \wedge \mathfrak{U}_{2}\right)^{\prime}$, $\left(\mathfrak{U}_{1} \wedge \mathfrak{U}_{2}\right)^{\prime \prime}$, then define $\sigma$ by:

$$
\begin{aligned}
\sigma= & \bar{\chi}\left(\left\ulcorner A_{1} \wedge A_{2}\right\urcorner\right) \cdot \sigma_{1}+\chi\left(\left\ulcorner A_{1} \wedge A\right\urcorner\right) \cdot \bar{\chi}\left(\left\ulcorner\left(\mathfrak{U}_{1} \wedge \mathfrak{U}_{2}\right)^{\prime}\right\urcorner\right) \cdot \sigma_{2} \\
& +\chi\left(\left\ulcorner A_{1} \wedge A_{2}\right\urcorner\right) \cdot \chi\left(\left\ulcorner\left(\mathfrak{U}_{1} \wedge \mathfrak{U}_{2}\right)^{\prime}\right\urcorner\right) \cdot \sigma_{3} .
\end{aligned}
$$

Other cases of $2^{\circ}$ are treated in a similar way.

$3^{\circ}$. For the extra formulas in ends $(Q)$, assign 0 to every denotation-variable. $4^{\circ}$ and $5^{\circ}$. Follow Section 2.

\section{§4. Some Variations}

1. We shall first discuss how to modify our formulation of the reduction of a 
formal system of intuitionistic arithmetic, which we shall call $\mathfrak{\Im} . \Im$ is obtained from $\mathfrak{N}$ by regarding $\vee, \supset$ and $\exists$ as primitive symbols and by imposing the condition that in the succedent of a sequent there be at most one sequent-formula. The definitions and the theorems we have established for $\mathfrak{N}$ are valid for $\mathfrak{\Im}$ also; only a minor adjustment is necessary. We shall note how to modify some notions.

In Definition 1.2, the formulas $\mathfrak{A}$ do not remain in the succedents of reducts and several cases be added: $\mathfrak{A}$ is $\mathfrak{B} \vee \mathfrak{C}, S$ is $\Gamma \rightarrow \mathfrak{A}$ and $\Sigma=\{\Gamma \rightarrow \mathfrak{B}\}$ or $\Sigma=\{\Gamma \rightarrow \mathfrak{C}\} ; \mathfrak{A}$ is $\mathfrak{B} \vee \mathfrak{C}, S$ is $\Gamma_{1}, \mathfrak{A}, \Gamma_{2} \rightarrow \Theta$ and $\Sigma=\left\{\Gamma_{1}, \mathfrak{B}, \mathfrak{A}, \Gamma_{2} \rightarrow \Theta ; \Gamma_{1}, \mathfrak{C}, \mathfrak{A}\right.$, $\left.\Gamma_{2} \rightarrow \Theta\right\}$; $\mathfrak{U}$ is $\mathfrak{B} \supset \mathfrak{C}, S$ is $\Gamma \rightarrow \mathfrak{A}$ and $\Sigma=\{\mathfrak{B}, \Gamma \rightarrow \mathfrak{E}\} ; \mathfrak{U}$ is $\mathfrak{B} \supset \mathfrak{E}, S$ is $\Gamma_{1}, \mathfrak{U}$, $\Gamma_{2} \rightarrow \Theta$ and $\Sigma=\left\{\Gamma_{1}, \mathfrak{A}, \Gamma_{2} \rightarrow \mathfrak{K}\right.$; $\left.\mathfrak{C}, \Gamma_{1}, \mathfrak{A}, \Gamma_{2} \rightarrow \Theta\right\} ; \mathfrak{A}$ is $\exists x \mathfrak{F}(x), S$ is $\Gamma \rightarrow \exists x \mathfrak{F}(x)$ and $\Sigma=\{\Gamma \rightarrow \mathfrak{F}(s)\}$ for some closed term $s ; \mathfrak{A}$ is $\exists x \mathfrak{F}(x), S$ is $\Gamma_{1}, \exists x \mathfrak{F}(x), \Gamma_{2} \rightarrow \theta$ and $\Sigma=\left\{\Gamma_{1}, \mathfrak{F}(n), \mathfrak{A}, \Gamma_{2} \rightarrow \Theta ; n=0,1,2, \cdots\right\}$.

Note that many of the weakenings in the succedent employed in the proof of Theorem 1 are unnecessary for $\Im$ due to the restriction on the sequents.

In Definition 2.1, the notions of positive and negative occurrences are subjected also to those additional connectives and such notions be defined also for the $\exists$-quantifiers. In Definition 2.2, $\mathfrak{U}^{p}$ is obtained from $\mathfrak{A}$ by knocking off all the positive $\forall$-quantifiers and the negative $\exists$-quantifiers in $\mathfrak{A}$, then replacing the remaining bound variables by distinct eigenvariables. In defining positive forms, only the singleton assignment is allowed to any $\exists$-quantifier in $\mathfrak{U}^{p}$. $(\exists x \mathfrak{F}(x))^{p}$ is dealt with as (4) there. The negative forms can be defined in a manner dual to the positive forms, except that the restriction of the "singleton assignment" as stated above is not imposed here. The quantifier-free forms of a sequent $S$ are defined as in Definition 2.3; here multi-numbers of formulas are permitted in the succedent. Theorem 3 holds as it reads. Note that $\Re$ and $\mathfrak{T}$ are equivalent when confined to quantifier-free sequents,

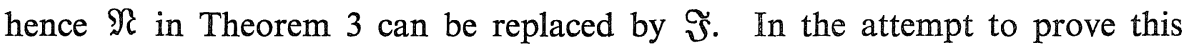
theorem for $\Im$, one finds that the singleton assignment to a $\exists$-quantifier in $\mathfrak{U}^{p}$ is adequate.

The proof of Theorem 1 for $\Im$ supplies us with the means to establish the well-known Harrop's result on intuitionistic arithmetic with regards to the connectives $\vee$ and $\exists$. This technique is originally due to Scarpellini (cf. [15]).

2. As an immediate consequence of Theorem 3 (both for $\mathfrak{N}$ and $\mathfrak{\Im}$ ) follows the theorem: a closed provable sequent whose antecedent-formulas are universal and succedent formulas are existential in prenex normal form is provable 
without essential cuts and inductions.

3. We shall next present a variation of the preceding results which regards some compound formulas (called elementary) as atomic. Related notions and results are seen in [16].

First introduce $<$ as a primitive symbol together with the axioms on it. A formula of thus extended $\mathfrak{R}$ is called elementary if all quantifiers occurring in it are bounded. A cut whose cut formula is elementary (an elementary cut) or an explicit logical inference whose principal formula is elementary except the $\forall$ in the succedent (an elementary logical inference) is regarded as a weak inference. The subsequent list shows how to modify the original results to the present purpose.

Definition 1.3, $(t)$. Read "closed, elementary formulas" in place of "closed equations."

Definition 1.4. The degree of a formula is defined to be the number of logical symbols in it relative to elementary formulas. (Elementary formulas have degree 0 .) The height and the ordinal assigned to a sequent are defined as usual relative to this notion of degree.

Notice that an elementary cut or an elementary logical inference does not increase the ordinal, since it is regarded as a weak inference.

In the proof of Theorem 1, introduce a new rule of inference, bounded quantification, as well as tr:

$$
b q \frac{\Gamma \rightarrow \Delta,(0<k \supset A(0)) \wedge \cdots \wedge(k-1<\mathbb{k} \supset A(k-1))}{\Gamma \rightarrow \Delta, \forall y(y<k \supset A(y))},
$$

where $A(b)$ is elementary and $k$ is a numeral. $(0<\mathbb{k} \supset A(0)) \wedge \cdots \wedge(k-1<$ $\mathbb{k} \supset A(k-1))$ is called the sub-formula of the $b q$ and $\forall y(y<k \supset A(y))$ the principal formula of the $b q$. The lower sequent is assigned the same ordinal as the upper sequent. The $b q$ is allowed only in the end-piece and is regarded as a weak inference.

$0^{\circ}$. $Q$ consists of closed elementary formulas, hence terminal. Stop.

$2^{\circ}$. Read as follows. The end-piece of $Q$ contains an ind. or an explicit logical inference whose principal formula is not elementary or which is an $\forall$ in the succedent.

2.2 . Case 1. $\forall x \mathfrak{F}(x)$ is not elementary. Do as in Section 1. Case 2. $\forall x \mathfrak{F}(x)$ is elementary, hence is of the form $\forall x<t R(x), R(0)$ being elementary. For the sake of simplicity, let us assume $\forall x \Im(x)$ remains unchanged to the endsequent. We quote the reduction for this case from [16]. 
Assume $\boldsymbol{t}=\boldsymbol{n}$ for some numeral $\boldsymbol{n}$. If $\boldsymbol{n}=0$, then $Q$ is reduced to

$$
\widetilde{\Delta}_{\Delta \rightarrow \Lambda_{1}, \forall x<t R(x), \Lambda_{2}}^{a<t \rightarrow} .
$$

If $\boldsymbol{n}>0$, consider the derivation $Q_{k}$ for each $k<\boldsymbol{n}$ :

$\because \because(k)$

$$
\begin{aligned}
& \alpha \\
& \Gamma \rightarrow \Theta, \mathbb{k}<\rrbracket \mathbb{R}(\mathbb{k}) \\
& \Gamma \rightarrow R<\pi \supset R(R), \widetilde{\theta, \forall x<t R(x)} \\
& \beta^{\circ} \\
& \Delta \rightarrow \mathbb{k}<n \supset R(k), \Lambda_{1}, \forall x<i R(x), \Lambda_{2}
\end{aligned}
$$

Then $Q$ is reduced to

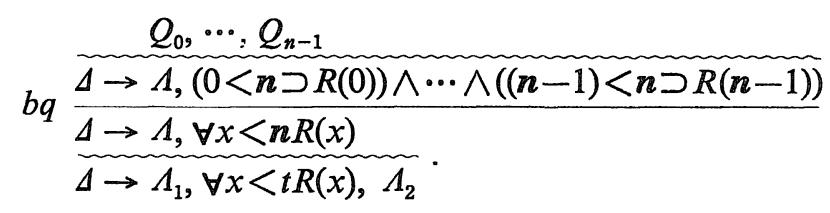

Since $\beta^{\prime}<\beta$, the ordinal decreases.

$3^{\circ}$. The elimination of weakenings in the end-piece is defined as before. If the auxiliary formula of an explicit logical inference or a $b q$ remains uneliminated, then apply that inference at the end.

$4^{\circ}$. If both occurrences of $\mathfrak{D}$ are explicit, either $Q$ consists of $\mathfrak{D} \rightarrow(\mathfrak{D}$ alone or all the formulas in $Q$ are elementary. $Q$ is terminal either case. Suppose $\mathfrak{D}$ is of the form

$$
\begin{aligned}
& \mathfrak{D} \rightarrow \mathfrak{D}
\end{aligned}
$$

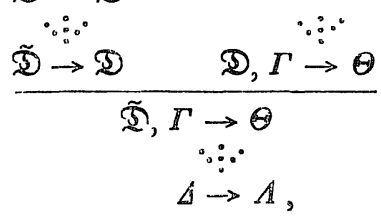

and some weak inferences apply to $\mathscr{D}$ to result in $\mathfrak{\mathfrak { D }}$. $Q$ is reduced to

$$
\begin{aligned}
& \text { (D) } \Gamma \stackrel{\because}{\because} \rightarrow \\
& \text { (⿹) } \stackrel{\because}{\because} \stackrel{\circ}{\rightarrow} \Theta \\
& \Delta \stackrel{\because}{\because},
\end{aligned}
$$

$5^{\circ}$. We may assume as in Section 1 that $Q$ properly contains its end-piece, 
and the existence of a suitable cut can be established as usual, hence the applicability of the essential reduction.

Finally, the $b q$ can be eliminated without essential cuts and inductions (cf. [16]); thus follows our theorem.

What about Theorem 3? To make the discussion simple, we deal with the "elementary sequents" only: the antecedent formulas are prenex universal in elementary formulas and the succedent formulas are prenex existential in elementary formulas. Define negative forms for the former and positive forms for the latter, leaving the unbounded $\forall$-quantifiers untouched. Hence there is no need to knock off any quantifiers and no necessity of eigenvariables. In (3) of 2), Definition 2.2, $l=r=1$. The denotation variables come in only at the prenex quantifiers. Theorem 3 can be stated and established as in Section 2: Let

$$
\begin{aligned}
- & \forall \mathfrak{X}_{1} A_{1}\left(\mathfrak{X}_{1}\right), \cdots, \forall \mathfrak{x}_{m} A_{m}\left(\mathfrak{x}_{m}\right) \\
\rightarrow & \exists \mathfrak{Y}_{1} B_{1}\left(\mathfrak{y}_{1}\right), \cdots, \exists \mathfrak{Y}_{n} B_{n}\left(\mathfrak{y}_{n}\right)
\end{aligned}
$$

be a provable elementary (and closed) sequent of $\mathfrak{N}$, where $\forall \mathfrak{x}$ is short for $\forall x_{1} \forall x_{2} \cdots \forall x_{k}$. Then there exist closed terms $\mathfrak{G}_{1,1}, \cdots, \mathfrak{g}_{1, p_{1}}, \cdots, \mathfrak{I}_{m, 1}, \cdots, \mathfrak{S}_{m, p_{m}}$, $\mathrm{t}_{1,1}, \cdots, \mathrm{t}_{1, q_{1}}, \cdots, \mathrm{t}_{n, 1}, \cdots, \mathrm{t}_{n, q_{n}}$, so

$$
\begin{aligned}
& A_{1}\left(\mathfrak{g}_{1,1}\right), \cdots, A_{1}\left(\mathfrak{g}_{1, p_{1}}\right), \cdots, A_{m}\left(\mathfrak{g}_{m, 1}\right), \cdots, A_{m}\left(\mathfrak{g}_{m, p_{m}}\right) \\
\rightarrow & B_{1}\left(\mathrm{t}_{1,1}\right), \cdots, B_{1}\left(\mathrm{t}_{1, q_{1}}\right), \cdots, B_{n}\left(\mathrm{t}_{n, 1}\right), \cdots, B_{n}\left(\mathrm{t}_{n, q_{n}}\right)
\end{aligned}
$$

is provable in $\mathfrak{R}$ without essential cuts and inductions.

From this follows that a provable sequent as $(*)$ is provable without essential cuts and inductions.

4.* We close this section with an application of our technique to the $\omega$-consistency of arithmetic. The $\omega$-consistency has been proven in [20], [21] and [25], and Kreisel has shown in [22] that $\varepsilon_{1}$-induction (applied to a suitable arithmetic) is optimal for a proof of the $\omega$-consistency. Here we shall briefly outline our method, which does not diverge much from Kreisel's first approach. For the functional translation of formulas and ordinal recursive functionals, the reader should refer to [23] and [24] also.

We employ Kreisel's formulation of the $\omega$-consistency (cf. Appendix of

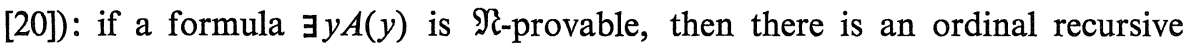

* This item has been added after G. Kreisel's suggestion. The author thanks him for his valuable comments on this article. 
functional $\Phi(p)$ (of some order) such that $p[\Phi(p)]$ is not (the number of) a proof in $\mathfrak{R}$ of the formula $>A(\Phi(p))$.

Definition 4.1. 1) Consider a closed sequent $S$ of $\mathfrak{R}$. If a positive occurrence $\forall y$ is in the scope of a negative occurrence $\forall x$ in a succedent formula $\mathfrak{A}$ of $S$, then $\forall y$ is said to depend on $\forall x$ in $\mathfrak{A}$. In an antecedent formula of $S$, the positive and the negative be reversed.

2) $\mathfrak{N}(\boldsymbol{f})$ will denote the system obtained from $\mathfrak{N}$ by adding to it some function parameters and new axioms and rules of inference concerning them. To make things simple, we describe the system for one unary function symbol $f(c f .[16])$.

(1) $f(m)=n_{1}, f(m)=n_{2} \rightarrow$

is an axiom, where $n_{1}$ and $n_{2}$ are distinct numbers. (f-axiom)

(2) f-replacement

$$
\frac{f(m)=n, \Gamma(n) \rightarrow \Delta(n)}{f(m)=n, \Gamma(f(m)) \rightarrow \Delta(f(m))}, \frac{f(m)=n, \Gamma(f(m)) \rightarrow \Delta(f(m))}{f(m)=n, \Gamma(n) \rightarrow \Delta(n)},
$$

where $\boldsymbol{m}$ and $\boldsymbol{n}$ are numerals and $\Gamma(n)$ and $\Delta(n)$ indicate some occurrences of $\boldsymbol{n}$ in $\Gamma$ and $\Delta$ respectively.

Proposition 4.1. Let $S: \mathfrak{N}_{1}, \cdots, \mathfrak{U}_{m} \rightarrow \mathfrak{B}_{1}, \cdots, \mathfrak{B}_{n}$ be a closed sequent of $\mathfrak{N}$. Let $\mathfrak{A}$ denote one of $\mathfrak{B}_{1}, \cdots, \mathfrak{B}_{n}\left(\mathfrak{A}_{1}, \cdots, \mathfrak{U}_{m}\right)$ and let $A(\boldsymbol{a}, \mathbb{Z})$ be the positive (negative) form of $\mathfrak{A}$ relative to the singleton assignment, where a denotes all the denotation variables and $\boldsymbol{b}$ denotes all the eigenvariables in $A$. Now, if $S$ is $\mathfrak{R}$ provable, then

$$
\begin{aligned}
& \left\{\forall x_{i} A_{i}\left(\boldsymbol{x}_{i}, \boldsymbol{f}_{i, 1}\left(\boldsymbol{x}_{i, 1}\right), \cdots, f_{i, k_{i}}\left(\boldsymbol{x}_{i, k_{i}}\right)\right)\right\}_{i} \\
\rightarrow & \left\{\exists y_{j} B_{j}\left(\boldsymbol{y}_{j}, \boldsymbol{g}_{j, 1}\left(\boldsymbol{y}_{j, 1}\right), \cdots, \mathfrak{g}_{j, l_{j}}\left(\boldsymbol{y}_{j, l_{j}}\right)\right\}_{j}\right.
\end{aligned}
$$

is $\mathfrak{N}(\boldsymbol{f})$-provable, where $\boldsymbol{f}$ 's and $\boldsymbol{g}$ 's are distinct function symbols, the $\boldsymbol{x}$ in a $\mathfrak{f}(\boldsymbol{x})$ represents all the bound variables such that the positive (negative) $\forall$ associated with $\boldsymbol{f}(\boldsymbol{x})$ depends on the negative (positive) $\forall$ 's associated with $x, i$ ranges over $1, \cdots, m$ and $j$ ranges over $1, \cdots, n$. Furthermore, one can construct a derivation of (1) primitive recursively in a derivation of $S$.

Proposition 4.2. The reduction method in Section 1 applies to $\mathfrak{\Re ( f )}$ with a minor modification.

Let $S: \Gamma \rightarrow \Delta$ be a closed sequent.

$$
\boldsymbol{\Sigma}=\left\{\boldsymbol{f}\left(\boldsymbol{m}_{1}\right)=\boldsymbol{n}_{1}, \cdots, \boldsymbol{f}\left(\boldsymbol{m}_{k}\right)=\boldsymbol{n}_{k}, \Gamma \rightarrow \Delta ; n_{1}, \cdots, n_{k}=0,1,2, \cdots\right\}
$$




$$
\text { for some } m_{1}, \cdots, m_{k}
$$

is a reduct set of $S$.

The f-replacement in Definition 4.1 does not increase the ordinal.

If the upper sequent of an ind. is assigned the ordinal $\omega^{\alpha_{1}}+\cdots+\omega^{\alpha_{k}}$ and if the induction term contains $p$ occurrences of $\boldsymbol{f}$, then the lower sequent is assigned the ordinal $\omega$<smiles>[13CH3][13CH3]</smiles>
is assigned the ordinal $\alpha$ and if the term to be quantified has $p$ occurrences of $f$, then the lower sequent is assigned the ordinal $\alpha+1+p$.

The $V J$-reduction when $t$ contains $f$ assumes the following form $Q_{n}$ :

$$
\begin{aligned}
& \mathfrak{F}(a), \stackrel{\because \because \bullet}{\rightarrow} \Theta, \mathfrak{F}\left(a^{\prime}\right) \\
& \mathfrak{F}(0), \Gamma \rightarrow \Theta, \widetilde{F}(s(n)) \\
& f(m)=n, \Im(0), \Gamma \rightarrow \Theta, \Im(s(n)) \\
& \underbrace{f(m)=\pi(0), \Gamma \rightarrow \Theta, \mathcal{F ( s ( f ( m ) ) )}}_{f(m)=\pi, I M \rightarrow A}
\end{aligned}
$$

where $t$ is of the form $s(\boldsymbol{f}(\boldsymbol{m}))$ (cf. [16]). This is defined for every $n$; place $\left\{Q_{n}\right\}_{n}$ above $Q$.

$$
o\left(Q_{n}\right)=\omega \dot{0}^{\omega^{\omega_{1}+1+(p-1)}}<\omega \dot{ }^{\omega^{\omega_{1}+1+p}}=o(Q) .
$$

ends $\left(Q_{n}\right)$ is related to ends $(Q)$ by the definition immediately above.

The reduction for an explicit $\forall$ in the antecedent is carried out in a similar manner.

Corollary. Suppose a sequent of the form (1) in Proposition 4.1, which we write $\Pi \rightarrow \Lambda$ here, is $\mathfrak{\Re}(\boldsymbol{f})$-provable. Then

$$
\boldsymbol{h}_{1}\left(\boldsymbol{m}_{1}\right)=\boldsymbol{n}_{1}, \cdots, \boldsymbol{h}_{q}\left(\boldsymbol{m}_{q}\right)=\boldsymbol{n}_{q}, I I \rightarrow \Lambda
$$

is provable without essential cuts and inductions, where $\boldsymbol{h}_{1}, \cdots, \boldsymbol{h}_{q}$ are some function symbols from f's and g's in (1), $m_{1}, \cdots, m_{q}$ are some numbers, and such a derivation is obtained uniformly in $n_{1}, \cdots, n_{q}$.

For the sake of convenience, we assume henceforth all the primitive recursive functions in $\Re$.

Proposition 4.3. There is an ordinal recursive functional $\phi(f, x)$ such that 


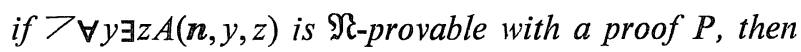

$$
>A(\boldsymbol{n}, \phi(\boldsymbol{f},\ulcorner P\urcorner), \mathfrak{f}(\phi(f,\ulcorner P\urcorner)))
$$

is verifiable for any specified interpretation of $f$. Here $A$ is quantifier free.

Proof. From the assumption we get a derivation of $\exists y>A(\boldsymbol{n}, y, \boldsymbol{f}(y))$ in $\mathfrak{N}(\boldsymbol{f})$ (Proposition 4.1). By the corollary of Proposition 4.2,

$$
\boldsymbol{f}\left(\boldsymbol{m}_{1}\right)=\boldsymbol{n}_{1}, \cdots, \mathfrak{f}\left(\boldsymbol{m}_{q}\right)=\boldsymbol{n}_{q} \rightarrow \exists y>A(\boldsymbol{n}, y, \mathfrak{f}(y))
$$

is provable without cuts and inductions for every $q$-tuple $\left(n_{1}, \cdots, n_{q}\right)$ (for some fixed $\left.m_{1}, \cdots, m_{q}\right)$. We can then find a number $m$ depending on $\left(n_{1}, \cdots, n_{q}\right)$ so that

$$
f\left(m_{1}\right)=n_{1}, \cdots, f\left(m_{q}\right)=n_{q} \rightarrow>A(n, m, f(m))
$$

is verfiable. For any specified interpretation of $f$, there is a $\left(n_{1}, \cdots, n_{q}\right)$ so $\boldsymbol{f}\left(\boldsymbol{m}_{1}\right)=\boldsymbol{n}_{1}, \cdots, \boldsymbol{f}\left(\boldsymbol{m}_{q}\right)=\boldsymbol{n}_{q}$ are true. $m$ is determined uniformly in $f$ and $\ulcorner P\urcorner$, thus can be expressed as $\phi(f,\ulcorner P\urcorner)$. This $\phi$ serves as the $\Xi$ in Lemma 1, p 47, of [20].

$\phi$ is defined uniformly in $f$ with the $\varepsilon_{0}$-recursion. The soundness of the system of such functionals can be established in primitive recursive arithmetic with the transfinite induction along $\varepsilon_{1}$. See [23] and [24].

\section{$§ 5$. Second Order Systems}

We can apply the reduction method to some second order systems of arithmetic. We assume the knowledge in the second order concepts. See [15] and [17] as references.

Second order free variables will be denoted by $\alpha, \beta, \cdots$, bound variables by $\varphi, \psi, \cdots$, and abstracts by $U, V, \cdots$. A second order system of arithmetic is obtained from $\mathfrak{N}$ by applying the inferences to all those sequents of the extended language and by adding the equality axioms

$$
s=t, \mathfrak{U}(s) \rightarrow \mathfrak{U}(t)
$$

for every formula $\mathfrak{A}$ as initial sequents and some rules of inference concerning second order quantifiers:

$\forall$ in the antecedent

$\frac{\mathfrak{F}(V), \Gamma \rightarrow \Theta}{\forall \varphi \mathfrak{F}(\varphi), \Gamma \rightarrow \Theta}$, where $V$ is an abstract.

$\forall$ in the succedent

$\frac{\Gamma \rightarrow \Theta, \mathfrak{F}(\alpha)}{\Gamma \rightarrow \Theta, \forall \varphi \mathfrak{F}(\varphi)}$, where $\alpha$ is an eigenvariable (viz., $\alpha$ does not occur in the 
lower sequent).

We do not specify the class of abstracts $V$ which are permitted in the $\forall$ in the antecedent.

The first system we shall consider, which we call $S$, is a second order system described as above, where the abstracts $V$ in the $\forall$ in the antecedent are restricted to arithmetical ones (namely, no second order quantifiers be involued in $V)$.

The second system we consider is SINN, the system with the $\Pi_{1}^{1}$-comprehension axiom which is formulated in [15].

1. It is known that $S$ can be interpreted by arithmetical predicates, or sets (cf. [17]). We can establish this fact by our technique.

Here closed sequents are those which have no free variables - first order or second order.

Two cases be added to Definition 1.2: $\mathfrak{A}: \forall \varphi \mathfrak{F}(\varphi), S: \Gamma \rightarrow \Theta_{1}, \mathfrak{N}, \Theta_{2}$ and $\Sigma=$ $\left\{\Gamma \rightarrow \Theta_{1}, \mathfrak{F}(V), \mathfrak{N}, \Theta_{2} ; V\right.$ ranges over all the closed, arithmetical abstracts $\}$; $\mathfrak{A}: \forall \varphi \mathfrak{F}(\varphi), S: \Gamma_{1}, \mathfrak{A}, \Gamma_{2} \rightarrow \Theta$ and $\Sigma=\left\{\Gamma_{1}, \mathfrak{F}(V), \mathfrak{A}, \Gamma_{2} \rightarrow \Theta\right\}$ for some closed, arithmetical $V$.

Let $\mathfrak{A}$ be a formula in a derivation $P$, let $l(A)$ denote the number of logical connectives in $\mathfrak{A}$ and let $\varepsilon(\mathfrak{U})$ be the sum of second order quantifiers in $\mathfrak{A}$ and second order eigenvariables in $\mathfrak{A}$. (We assume that a free variable is used as eigen only at one $\forall$ in the succedent and it occurs only in the related places.) Define $g(\mathfrak{A} ; P)$, the grade of $\mathfrak{A}$ relative to $P$, to be $\omega \cdot \varepsilon(\mathfrak{U})+l(\mathfrak{A})$.

Corollary. $\varepsilon(\mathfrak{\Im}(\alpha))=\varepsilon(\mathfrak{F}(V))+1$, hence

$$
g(\mathfrak{\mho}(V))<g(\mathfrak{\mho}(\alpha))
$$

if $\alpha$ is an eigenvariable and actually occurs in $F(\alpha)$ and $V$ is arithmetical not containing eigenvariables.

$$
g(\mathfrak{\Im}(V))<g(\forall \varphi \Im(\varphi))
$$

if $V$ satisfies the same condition as above.

It is convenient to use the system of ordinal diagrams $0\left(1, \omega^{2}\right)$ for the reduction of $S$ (cf. [17]): We write $(a, \alpha)$ for $(0, a, \alpha)$ here.

The ordinal diagram (o.d.) of an initial sequent is 0 .

A weak inference does not increase the o.d.

If $\alpha$ is the o.d. of the upper sequent of a logical inference with one upper 
sequent, then the o.d. of its lower sequent is $\sigma+1$. For one with two upper sequents, assign $\sigma_{1}$ 半 $\sigma_{2}$.

If the o.d.'s of the upper sequents of a cut are $\sigma_{1}$ and $\sigma_{2}$ and the grade of the cut formula is $g$, then the lower sequent is assigned the o.d. $\left(g, \sigma_{1}\right.$

If the $o . d$. of the upper sequent of an ind. is $\sigma$ and the grade of the induction formula is $g$, then its lower sequent is assigned the $o . d .(g+1, \sigma)$.

Let us write $<$ for $<_{0}$ of the system $O\left(1, \omega^{2}\right)$. For the computation of o.d.'s, consult [15] and [17].

$1^{\circ}$. If there is a non-eigen second order free variable in the endpiece of $Q$, then substitute for it the abstract $\left\{x_{1} \cdots x_{n}\right\}(0=0)$.

$2^{\circ}$. $I$ is a second order $\forall$ in the antecedent:

$$
\begin{gathered}
\mathfrak{F}(V), \Gamma \stackrel{\stackrel{\circ}{\rightarrow}}{\rightarrow} \Theta \\
\forall \varphi \mathfrak{F}(\varphi), \Gamma \stackrel{\sigma+1}{\rightarrow} \Theta \\
\Gamma_{1}, \forall \varphi \mathfrak{F}(\varphi), \Gamma_{2} \stackrel{\circ}{\rightarrow} \Theta^{\prime} .
\end{gathered}
$$

Note that $V$ is closed under the condition $1^{*}$. Define $Q^{\prime}$ :

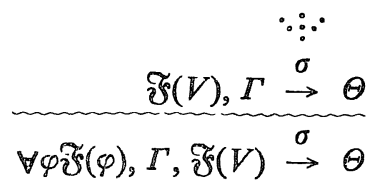

$$
\begin{aligned}
& \Gamma_{1}, \mathfrak{乛}(V), \forall \varphi \mathfrak{f}(\varphi), \stackrel{\because}{\dot{\Gamma}_{2}} \rightarrow \Theta^{\prime} .
\end{aligned}
$$

$I$ is a second order $\forall$ in the succedent:

$$
\begin{aligned}
& \because \\
& \stackrel{\sigma}{\sigma}(\alpha) \\
& \Gamma \stackrel{\rightarrow}{\rightarrow} \Theta, \mathfrak{F}(\alpha) \\
& \hline \Gamma \rightarrow \Theta, \forall \varphi \mathfrak{F}(\varphi) \\
& \stackrel{\because}{\rightarrow} \Theta_{1}, \forall \varphi \mathfrak{F}(\varphi), \Theta_{2} .
\end{aligned}
$$

For each closed, arithmetical $V$, define $Q_{V}$ :

$$
\begin{aligned}
& \because \because(V) \\
& \Gamma \stackrel{\sigma^{\prime}}{\rightarrow} \Theta, \mathfrak{F}(V)
\end{aligned}
$$

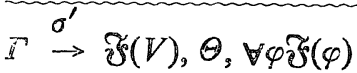

$$
\begin{aligned}
& \Gamma^{\prime} \stackrel{\because}{\rightarrow} \Theta_{1}, \mathfrak{\Im}(V), \forall \varphi \Im(\varphi), \Theta_{2} .
\end{aligned}
$$


$\sigma^{\prime} \leq \sigma$ since the substitution of $V$ for $\alpha$ does not increase the grade. (See Corollary above.)

Between $2^{\circ}$ and $3^{\circ}$, insert the reduction of an equality axiom in the end-piece. (See [15] and [17].)

$5^{\circ}$. The case where the suitable cut concerns the second order inferences. The reduction is done in the same manner as 10.1.2 of Chapter 2, [15]. Note that the comprehension abstract $V$ is closed and arithmetical, hence the decrease of o.d.'s.

2. An interpretation of SINN in a system with the constructive $\omega$-rule has been given in [16]. Here a closed formula or a closed sequent means one closed with respect to first order variables; hence it allows second order parameters.

In Definition 1.2, add two cases: $\mathfrak{A}: \forall \varphi \mathfrak{F}(\varphi), S: \Gamma \rightarrow \Theta_{1}, \mathfrak{A}, \Theta_{2}$ and $\Sigma$ $=\left\{\Gamma \rightarrow \Theta_{1}, \mathfrak{F}(V), \mathfrak{A}, \Theta_{2} ; V\right.$ ranges over all the semi-isolated (or, $\Pi_{1}^{1}$ - in wider sense) closed abstracts $\} ; \mathfrak{A}: \forall \varphi \mathfrak{F}(\varphi), S: \Gamma_{1}, \mathfrak{A}, \Gamma_{2} \rightarrow \Theta$ and $\Sigma=\left\{\Gamma_{1}, \mathfrak{F}(V), \mathfrak{A}\right.$, $\left.\Gamma_{2} \rightarrow \Theta\right\}$, for some semi-isolated closed abstract $V$. With a technique which is a combination of the reduction methods in 1 of this article and in [16], we can establish our Theorem 1 for SINN.

3. Although this is not strictly along the line of this article, let us remark on the $\omega$-consistency. Let $\mathfrak{S}$ denote any system of arithmetic which has an effective translation into a system of constructive $\omega$-rule, say $\mathfrak{S}^{*}$, which accepts a constructive cut elimination proof, hence a consistency proof. $\subseteq$ can be first order arithmetic (cf. [12]), the $\mathfrak{S}$ in 1 of this section, SINN (cf. [15], [16] and [26]), or the system with the provably $\Delta_{2}^{1}$-comprehension axiom (cf. [27]).

Suppose $\exists x \mathfrak{F}(x)$ and $7 \mathfrak{F}(\boldsymbol{m})$ are $S$-provable for all $m$, where there is an effective enumeration of derivations of $7 \mathfrak{F}(\boldsymbol{m})$ for all $m$. Then $\exists x \mathfrak{F}(x)$ and $\forall x>\mathfrak{F}(x)$ are $S^{*}$-provable, contradicting the consistency of $\mathfrak{S}^{*}$.

A constructive version of the $\omega$-consistency for any such system requires a more delicate analysis of the system.

\section{References}

[1] Gentzen, G., Die Widerspruchsfreiheit der reinen Zahlentheorie, Math. Ann., 112 (1936), 493-565.

[2] —, Neue Fassung des Widerspruchsfreiheitsbeweises für die reine Zahlentheorie, Forschungen zur Logik und zur Grundlegung der exakten Wissenschaften, New Series, 4, Leipzig (1938),_19-44. 
[3] - Beweisbarkeit und Unbeweisbarkeit von Anfangsfällen der transfiniten Induktion in der reinen Zahlentheorie, Math. Ann., 119 (1943), 140-161.

[4] Gödel, K., Über eine bisher noch nicht benützte Erweiterung des finiten Standpunktes, Bibliotheque Scientifique, 34 Logica (1960), 76-83.

[5] Kino, A., On provably recursive functions, J. Math. Soc. Japan, 20 (1968), 456-476.

[6] Kreisel, G., On the interpretations on non-finitist proofs-Part I, J. Symbolic Logic, 16 (1951), 241-267.

[7] —, On the interpretations of non-finitist proofs-Part II, ibid., 17 (1952), 43-58.

[8] —, Mathematical significance of consistency proofs, ibid., 23 (1958), 155-182.

[9] — Proof by transfinite induction and definition by transfinite induction in quantifier-free systems, ibid., 24 (1959), 322-323.

[10] - Interpretations of analysis by functionals, Constructivity in Mathematics, North-Holland Publishing Co., Amsterdam (1959).

[11] - A survey of proof theory, J. Symbolic Logic, 33 (1968), 321-388.

[12] Schütte, K., Beweistheorie, Springer (1960).

[13] Szabo, M. E., Editor, The Collected papers of Gerhard Gentzen, North-Holland Publishing Co., Amsterdam and London (1969).

[14] Tait, W. W., A characterization of ordinal recursive functions, J. Symbolic Logic, 24 (1959), 325.

[15] Takeuti, G., Consistency proofs of subsystems of classical analysis, Ann. Math., 86 (1967), 299-348.

[16] - The $\Pi_{1}^{1}$-comprehension schema and $\omega$-rules, Proc. of the Summer School in Logic 1967, Lecture notes in Mathematics, 70 (1968), 303-330.

[17] —, Proof Theory, North-Holland Publishing Co., Amsterdam, Oxford and New York (1975).

[18] Takeuti, G. and Yasugi, M., Reflection principles of subsystems of analysis, Contributions to Mathematical Logic, edited by K. Schütte, North-Holland Publishing Co., Amsterdam (1968), 255-273.

[19] —, Sûgaku Kisoron (in Japanese), Kyôritsu Publishing Co., Tokyo (1974).

\section{Added in References}

[20] Kreisel, G., Models, translations and interpretations, Mathematical interpretations of formal systems, edited by Th. Skolem et al., North-Holland Publishing Co., Amsterdam (1955), 26-50.

[21] Parsons, C., The $\Omega$-consistency of ramified analysis, Archiv für mathematische Logik und Grundlagenforschung, 6 (1962), 30-34.

[22] G. Kreisel, The review of [21], Zbl. Math., 106 (1964), 238-239.

[23] Tait, W. W., Functionals defined by transfinite recursion, J. Symbolic Logic, 30 (1965), 155-174.

[24] - The substitution method, ibid., 30 (1965), 175-192.

[25] Kreisel, G. and Lévy, A. Reflection principles and their use for establishing the complexity of axiomatic systems, Zeitschrift für mathematische Logik und Grundlagen der Mathematik, 14 (1968), 97-142.

[26] Yasugi, M., Cut elimination theorem for second order arithmetic with the $\Pi_{1}^{1}$-comprehension axiom and the $\omega$-rule, J. Math. Soc. Japan, 22 (1970), 308-324.

[27] Takeuti, G., and Yasugi, M., The ordinals of the systems of second order arithmetic with the provably $\Delta_{2}^{1}$-comprehension axiom and with the $\Delta_{2}^{1}$-comprehension axiom respectively, Japan. J. Math., 41 (1973), 1-67. 
